\title{
The Circadian Pacemaker in the Aplysia Eye Sends Axons Throughout the Central Nervous System ${ }^{1}$
}

\author{
LEAH M. OLSON ${ }^{2}$ AND JON W. JACKLET \\ Neurobiology Research Center, Department of Biological Sciences, State University of New York at Albany, Albany, New York 12222
}

\begin{abstract}
Each eye of Aplysia contains a population of electrically coupled pacemaker neurons whose synchronous activity can be recorded from the optic nerve as a compound action potential (CAP). The CAP frequency continues to show a circadian rhythm even when the eye is isolated from the animal and maintained in constant conditions, and thus it contains an autonomous circadian pacemaker, which may reside in the pacemaker neurons. The pacemaker neurons, along with retinal photoreceptors, send axons out of the optic nerve, which connects to the cerebral ganglion of the central nervous system (CNS). Pacemaker neurons, but not photoreceptors, may contain an aminergic transmitter, possibly dopamine (DA). We describe the central projections of optic nerve fibers using horseradish peroxidase filling of the cut optic nerve, and transport of radiolabeled macromolecules after selective exposure of the eye to $\left[{ }^{3} \mathrm{H}\right]$ leucine, which labels both pacemaker neurons and photoreceptors. We were able to determine the projections of pacemaker axons by exposing the eye to $\left[{ }^{3} \mathrm{H}\right]-3,4$-dihydroxyphenylalanine $\left({ }^{3} \mathrm{H}\right]$ DOPA and $\left[{ }^{3} \mathrm{H}\right] \mathrm{DA}$, which is preferentially taken up and transported by the pacemaker neurons. Pacemaker axons project bilaterally to the cerebral, pedal, and pleural ganglia and may extend as far as the abdominal ganglion. We corroborate this anatomical evidence by recording an orthodromic CAP in the optic nerve that had originated in the eye and subsequently recording the CAP in the CNS connectives and nerves that contained $\left[{ }^{3} \mathrm{H}\right]$ DOPA-labeled fibers. These results suggest that circadian pacemaker information from the eye is widely distributed throughout the CNS, including neural structures known from studies by others to mediate circadian-regulated behaviors, such as locomotion. Thus, Aplysia can now be used as a model system to examine the influence of the central projections of an identified circadian pacemaker on behavior, such as locomotion, at the level of identified central neurons.
\end{abstract}

The circadian system influences behavior by imposing rhythmicity and internal temporal order on a myriad of physiological and behav-

Received March 18, 1985; Revised May 28, 1985;

Accepted May 30, 1985

${ }^{1}$ We wish to thank Suzanne Tieman for her help and suggestions in the completion of this study, and for her critical reading of the manuscript. We would also like to thank Ashraf Azab for providing confirmation of some of the electrophysiological data. This work was supported by National Science Foundation Grant BNS $82-06245$ to J. W. J.

${ }^{2}$ To whom correspondence should be sent, at her present address: Department of Psychology, Oberlin College, Oberlin, $\mathrm{OH} 44074$. ioral functions through an endogenous oscillator within the system, the circadian pacemaker. The coordination and timing of these internal rhythms with important environmental periodicities, primarily the light/dark cycle, is brought about by entrainment of the circadian pacemaker.

Conceptually and functionally, the circadian system can be divided into three components: $(1)$ the circadian pacemaker, which generates a self-sustaining, endogenous rhythm with a period close to $24 \mathrm{hr}$; (2) input pathways to the pacemaker that carry environmental information that entrains the pacemaker; and (3) outputs from the pacemaker, which carry timing information to effector systems.

Formal properties of circadian rhythms have been developed by analyzing the rhythmic behavioral and physiological processes that are driven by the pacemaker (Pittendrigh and Daan, 1976; Aschoff, 1981; Pittendrigh, 1981). In these studies the pacemaker system itself is often an unknown anatomical entity. The inputs to the system are manipulated and the subsequent perturbations to the pacemaker are inferred from measurements of the driven rhythmic outputs. This approach has resulted in a number of useful formal models of the circadian system, but further understanding of the function and mechanism of the circadian system ultimately depends on a more precise knowledge of its anatomical and physiological substates.

Recent work aimed at defining the anatomical organization of these components has been most successful in localizing the pacemakers themselves. Within the nervous system of a variety of species discrete loci now have been identified that possess an endogenous, self-sustaining pacemaker capable of directing the rhythmicity of effector systems and secondary oscillators (for reviews see Page, 1981; Menaker and Binkley, 1981; Jacklet, 1984, 1985). The successful anatomical localization of circadian pacemakers has allowed research on the cellular mechanisms of the pacemaker (see Jacklet, 1984) and the process of entrainment (Eskin, 1979; Rusak, 1979; Zatz, 1979), and has sharpened the conceptual framework used to address further questions concerning the organization of the circadian system. An important conclusion from these studies is that a single (functional) pacemaker can control rhythmicity in widely dissimilar effector systems, suggesting that the output from the pacemaker must be extensive and complex.

${ }^{3}$ The abbreviations used are: ASW, artificial seawater, AT, anterior tentacle; $\mathrm{CAP}$, compound action potential; $\mathrm{CM}$, culture medium; CNS, central nervous system; C.Pd, cerebral-pedal (connective); C-PI, cerebral-pleural (connective); DA, dopamine; DAB, diaminobenzidine; DOPA, 3,4-dihydroxyphenylalanine; HRP, horseradish peroxidase: 5-HTP, 5-hydroxytrytophan: MCG, metacerebral giant neuron; NE, norepinephrine; PI-Ab, pleural-abdominal (connective); PT, posterior tentacle; SCN, suprachiasmatic nuclei; ULAB, upper lip (nerve). 
Characterizing the output pathways from an identified pacemaker to its effector systems has been difficult. The mammalian brain structures identified as primary circadian pacemakers, such as the suprachiasmatic nuclei $(\mathrm{SCN})^{3}$ of the hypothalamus, are complex (for review see Takahashi and Zatz, 1982; Moore, 1983). Whereas many of the anatomical pathways from the SCN have becn identified recently (Moore, 1983), the pathways that carry the pacemaker information are unknown, in part because the specific subpopulations of neurons within the SCN that function as the pacemakers have not yet been identified.

The circadian pacemaker system of Aplysia is well suited for a model system to describe the output pathways of a pacemaker. It has an identified, autonomous circadian pacemaker in each eye (Jacklet, 1969); the output of the pacemaker is a rhythm in frequency of a distinctive compound action potential (CAP) recorded from the optic nerve; the neuronal population that generates the output signal of the pacemaker has been identified (Jacklet et al., 1982); and a single output pathway, the optic nerve, connects the ocular pacemakers to the brain. In this study we describe the anatomical pathways within the brain from the circadian pacemaker in the Aplysia eye using horseradish peroxidase (HRP) filling of the cut optic nerve and transport of radiolabeled compounds from the eye to the CNS. We also present electrophysiological evidence for pacemaker activity in those pathways. We find that pacemaker neurons from each retinal circadian pacemaker project bilaterally throughout virtually the entire nervous system. Preliminary reports of these findings have appeared in abstract form (Olson and Jacklet, 1982, 1983).

\section{Materials and Methods}

Aplysia californica (75 to $150 \mathrm{gm}$ ) were purchased from Pacific Biomarine (Venice, CA), Sea Life Supply (Sand City, CA), or Alacrity Marine (Redondo Beach, CA), housed in instant Ocean aquaria at $15^{\circ} \mathrm{C}$, and maintained on a light/dark cycle of $13 \mathrm{hr}$ light/11 $\mathrm{hr}$ dark. Excised nervous tissue was maintained in either artificial seawater (ASW: $451 \mathrm{~mm} \mathrm{NaCl}, 10 \mathrm{~mm} \mathrm{KCl}, 10$ $\mathrm{mm} \mathrm{CaCl}_{2}, 22 \mathrm{~mm} \mathrm{MgCl}_{2}, 2.5 \mathrm{~mm} \mathrm{NaHCO}, \mathrm{pH} 7.8$ ) or culture medium (CM) as described by Jacklet (1977) except that the dextrose concentration was reduced to $10 \mathrm{mM}$ and the amino acids mixture was reduced from $10 \%$ to $1 \%$. in $\mathrm{CM}$, eyes can remain viable for up to 2 weeks. Any changes to these basic formulae are given in individual experiments.

HRP tracing experiments. The projections of optic nerve fibers into the brain were stained using the HRP technique. Cerebral ganglia with attached eyes and with or without attached pedal and pleural ganglia were dissected and placed in $\mathrm{CM}$ made up without $\mathrm{CaCl}_{2}\left(0 \mathrm{Ca}^{21} \mathrm{CM}\right)$. The connective tissue sheath surrounding the ganglia was carefully thinned and the optic nerve was freed from other nerve trunks close to the ganglion. Ganglia were transferred to an incubation dish containing a glass micropipette with a tip pulled to the diameter $(75$ to $100 \mu \mathrm{m})$ of the nerve. The optic nerve was cut $1 \mathrm{to} 2 \mathrm{~mm}$ from the cerebral ganglion and drawn into the tip of the pipette with gentle suction. The CM was removed from the shaft of the pipette with a Hamilton syringe and replaced with 1 to $2 \mu$ l of a $20 \%$ HRP solution (20\% HRP, Sigma type VI, and $3 \% \alpha$-lysophosphatidyl choline from egg albumin (Sigma Chemical Co., St. Louis, MO) in distilled water). The incubation dish was covered and stored at $15^{\circ} \mathrm{C}$ for 9 to $24 \mathrm{hr}$ to allow transport of the HRP. Ganglia were fixed in 1\% paraformaldehyde and $2.5 \%$ glutaraldchyde in $2 \%$ sym-collidine buffer, $\mathrm{pH} 7.2$, with $0.7 \mathrm{M}$ sucrose and $0.75 \mathrm{~mm} \mathrm{CaCl}_{2}$, for 4 to $24 \mathrm{hr}$. After thorough rinsing, the preparation was preincubated in $10 \mathrm{ml}$ of $0.05 \%$ diaminobenzidene (DAB; Sigrna, grade II) in $0.1 \mathrm{M}$ phosphate buffer, $\mathrm{pH} 6.6$, with $0.7 \mathrm{M}$ sucrose, on ice overnight. The following morning, 6 to 10 drops of $0.3 \% \mathrm{H}_{2} \mathrm{O}_{2}$ were added to the DAB incubation solution, and the tissue was monitored under a dissecting microscope for tormation of reaction product. The reaction was terminated after about $50 \mathrm{~min}$ by rinsing in phosphate buffer. For light microscope processing, ganglia were rinsed, dehydrated in a graded ethanol series, cleared in xylene, and either whole mounted on a depression slide or embedded in paraffin for sectioning. Ganglia processed for ultrastructural studies were cut in half and postfixed in $2 \%$ osmium tetroxide in $0.2 \mathrm{M} \mathrm{sym}$-collidine buffer with $0.7 \mathrm{M}$ sucrose for 1 to $2 \mathrm{hr}$, rinsed in buffer with sucrose, dehydrated in ethanol and propyline oxide, and cmbedded in Mollenhauer's Epon/Araldite mixture II (Mollenhauer, 1964) before $5-\mu \mathrm{m}$ serial sections were cut on an Ultramicrotome. When
HRP was identified in a section, the block was retrimmed, and five to seven ultrathin serial sections (silver to light gold interference colors) were taken from the area of interest. Sections were placed on Formvar-coated, $600-\mu \mathrm{m}$ single-hole grids (Fullam, Schenectady, NY), and selected grids from each series were post-stained with lead citrate (Reynolds, 1963) and $2 \%$ uranyl acetate in methanol. In all cases, stained and unstained sections from each series were examined to confirm identification of the DAB reaction product, which was most easily identified in unstained material.

Autogradiographic studies. To trace optic afferents in the ganglia, eyes were selectively exposed to a tritiated leucine solution, and axons within the CNS were labeled by transport of incorporated leucine and visualized by autoradiography. Previous work suggests that pacemaker neurons may utilize an aminergic transmitter, possibly dopamine (Luborsky-Moore and Jacklet, 1976b, 1977); therefore, similar studies used tritiated transmitters and precursors $\left(\left[{ }^{3} \mathrm{H}\right]-3,4\right.$-dihydroxyphenylalanine (DOPA), $\left[{ }^{3} \mathrm{H}\right]$ dopamine (DA), $\left[{ }^{3} \mathrm{H}\right]$ norepinephrine (NE), and $\left[{ }^{3} \mathrm{H}\right]-5$-hydroxytryptophan $\left.(5-\mathrm{HTP})\right)$ to selectively identify the pathways of pacemaker neurons. Work in a related mollusc, Helix, suggests that precursors may be more readily accumulated by the soma membrane, whereas the transmitter itself is specifically accumulated by terminals (Turner and Cottrell, 1978).

The following labeled compounds were dried down under vacuum and resuspended in the appropriate incubation solution (see below): $\mathrm{L}-\left[4,5{ }^{3} \mathrm{H}(\mathrm{N})\right]$ leucine, $58 \mathrm{Ci} / \mathrm{mmol}\left(\mathrm{ICN}\right.$, Irvine, CA); $\left[{ }^{3} \mathrm{H}\right] \mathrm{DOPA}\left(L-\left[3,4 \cdot{ }^{3} \mathrm{H}(\mathrm{G})\right]\right.$ dihydroxyphenylalanine), $32.2 \mathrm{Ci} / \mathrm{mmol}$ (New England Nuclear, Boston, MA); [ $\left.{ }^{3} \mathrm{H}\right] \mathrm{NE}$ (DL- $\left\{7^{3} \mathrm{H}(\mathrm{N})\right]$ norepinephrine hydrochloride, $14.4 \mathrm{Ci} / \mathrm{mmol}$ (New England $\mathrm{Nu}$ clear): $\left[{ }^{3} \mathrm{H}\right]-5-\mathrm{HTP}\left(\mathrm{DL}-5\right.$-hydroxy-[G-$\left.{ }^{3} \mathrm{H}\right]$ tryptophan), $2.5 \mathrm{Ci} / \mathrm{mmol}$ (Amersham Corp., Arlington Heights, IL); $\left[{ }^{3} \mathrm{H}\right] \mathrm{DA}\left(3,4-\left[\right.\right.$ ring-2,5,6- $\left.{ }^{3} \mathrm{H}\right]$ dihydroxyphenylethylamine hydrochloride), $41.7 \mathrm{Ci} / \mathrm{mmol}$ (New England Nuclear). Eyes or ganglia to be labeled were exposed to the tritiated compound $\left(1 \times 10^{-7}\right.$ to $4 \times 10^{-6}$ M) for 6 to $8 \mathrm{hr}$. For tracing experiments one eye of each preparation was isolated from the brain by a Vasoline seal around the optic nerve in a twochambered dish. The solution in the cyo chamber was replaced with $250 \mu$ of a tritiated incubation medium. For labeling with $\left[{ }^{3} \mathrm{H}\right]$ leucine, eyes were incubated in ASW with $10 \mathrm{~mm}$ HEPES buffer, $10 \mathrm{~mm}$ dextrose, and 10,000 units of penicillin/streptomycin and vitamins containing $20 \mu$ of $\left.{ }^{3} \mathrm{H}\right]$ leucine/ $250 \mu$ l of incubation medium (final concentration, $1 \times 10^{-6} \mathrm{M}$ ) for 2 to $6 \mathrm{hr}$ and washed for 30 to $60 \mathrm{~min}$ in cold medium; then the preparation was placed in fresh ASW for 6 to $36 \mathrm{hr}$ to allow transport of incorporated material For labeling with tritiated transmitters and precursors, $1 \mathrm{~mm}$ ascorbic acid and $1 \times 10^{-5} \mathrm{M}$ pargyline (Sigma) were added to the incubation medium and the HEPES buffer was increased to $30 \mathrm{~mm}$. The solution was titrated to $\mathrm{pH}$ 7.8 with $\mathrm{NaOH}$. Eyes were maintained in the labeling medium for the entire transport time of $24 \mathrm{hr}$. After labeling, the tissue was fixed, rinsed, and dehydrated. Before clearing in xylene and paraffin embedding, the individual ganglia (cerebral, pleural, pedal, and abdominal) were cut away from each other. A long piece ( 1 to $1.5 \mathrm{~mm}$ ) of pleural-abdominal (PI-Ab) connective (see Fig. 1) was left attached to both pleural and abdominal ganglia to facilitate its identification after sectioning. Twelve-micrometer sections were cut and attached to subbed slides. After defatting in xylene and rehydration, prepared slides were dipped in a mixture of 1:1 $\mathrm{NTB}_{2}$ nuclear track emulsion (Eastman-Kodak, Rochester, NY) and distilled water, and packaged in lighttight boxes containing dessicant. Slides were stored at $4^{\circ} \mathrm{C}$ for 4 to 6 weeks, developed in Kodak D-19 developer, fixed in Kodak fixer, and post-stained with azure-eosin. In some cases, tissue was processed in epoxy; $5-\mu \mathrm{m}$ sections were dried onto untreated slides, dipped, and processed as above.

Electrophysiology. The presence of CAP activity, which represents the synchronous firing of the retinal pacemaker neurons (Jacklet et al., 1982), was looked for in CNS pathways shown in anatomical studies to receive photoreceptor or pacemaker fibers, by making extracellular recoidings, using polyethylene tubing suction electrodes, simultaneously from the optic nerve (en passant) and those connectives or nerves. Head ganglia with eyes attached were dissected and pinned in a Sylgard-bottomed recording dish containing ASW or CM. Electrodes were led to a switch box that allowed selection of a stimulate or record mode. Electrical stimulation was delivered with a W-P Instruments (New Haven, CT) Anapulse Stimulator. Recordings were amplified and displayed on a dual beam storage oscilloscope. To examine the temporal relationship between connective potentials and optic nerve potentials, the spontaneous orthodromic CAPs or electrical stimulation of nerves and connectives were used to trigger the oscilliscope trace. Photographs of the oscilliscope trace were taken after repeated sweeps with the trace intensity turned down to emphasize potentials in the connectives with exact temporal relation to the optic nerve CAP. Recordings were obtained from dark-adapted, dimly illuminated preparations. Under these conditions, the rate of spontaneous, pacemaker CAPS is slightly higher than that occurring in constant darkness 


\section{Results}

The CNS of Aplysia (Fig. 1) consists of four paired head ganglia which form a ring around the esophagus, and a single, unpaired, caudally located abdominal ganglion. Each eye projects to the ipsilateral cerebral hemiganglion via a long (1 to $2 \mathrm{~cm}$ ) optic nerve, which contains the axons of about 3000 photoreceptors and about 1000 pacemaker neurons (Jacklet et al., 1982).

\section{HRP tracing of optic nerve fibers}

Previous attempts to trace optic nerve fibers into the cerebral ganglion using cobalt chloride fiber tracing were only partially successful (Luborsky-Moore and Jacklet, 1976a; Jahan-Parwar and Fredman, 1976), probably due to the small diameter $(0.2$ to $0.6 \mu \mathrm{m})$ of optic fibers. Tracing studies by Luborsky-Moore and Jacklet (1976a) identified a population of efferent neurons in the ipsilateral cerebral hemiganglion that sends axons to the eye through the optic nerve, but the bulk of optic fibers, presumably the afferents, were traced only a short distance into the ganglion. In the present study, fibers labeled with HRP could be traced for much greater distances than could those in cobalt studies. The following description is based on the combined results from $>20$ preparations, which varied in the extent of filling. No differences were seen between the projections from the left or right eye, which form mirror-image projections in each cerebral hemiganglion.

Most of the HRP-filled optic fibers are afferent fibers of very fine caliber ( 0.2 to $0.6 \mu \mathrm{m}$ diameter), but each nerve contains a small number of larger, efferent fibers ( 1 to $3 \mu \mathrm{m}$ diameter). Each optic nerve enters the ipsilateral cerebral hemiganglion dorsally and rostrally, parallel and lateral to the posterior tentacle (PT) nerve, and filled fibers extend as a coherent optic tract caudally within the hemiganglion (Fig. 2A), where they separate into several distinct tracts (Fig. $2 B$ ). One trace consists of large-diameter fibers that can be followed rostrally to about 13 HRP-filled neurons located either

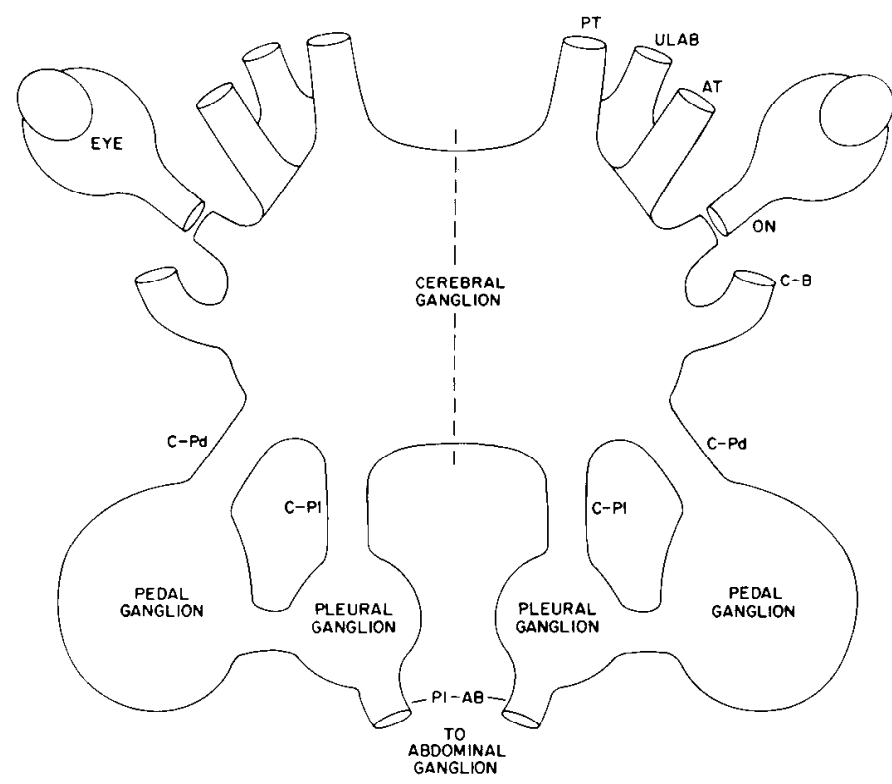

Figure 1. Schematic drawing of the CNS of Aplysia. Rostral is toward the top of the figure. The cerebral ganglion $(2500 \times 1500 \mathrm{~mm})$ is an embryonically fused pair of bilaterally symmetrical ganglia that receive the bulk of sensory input from the head. Eyes are altached by the optic nerves (ON), shown cut here. Head nerves shown are the posterior tentacle $(P T)$, anterior tentacle $(A T)$, and upper lip (ULAB) nerves. The cerebral gangion is attached to the buccal ganglion (not shown) by the cerebral-buccal $(C-B)$ connectives and to the paired pedal and pleural ganglia by the cerebral-pedal $(C-P d)$ and cerebral-pleural (C-PI) connectives, respectively. These head ganglia form a ring around the esophagus. The pleural-abdominal $(P I-A b)$ connectives attach the pleural ganglia to the caudally located abdominal ganglion (not shown). along the ipsilateral PT nerve in rostral ganglion or embedded within the PT nerve some distance beyond the ganglion (Fig. 2, $A$ and $C$ ). These neurons appear identical to the efferent neurons described in cobalt tracing studies (Luborsky-Moore and Jacklet, 1976a). The small caliber afferent fibers take either a medial trajectory toward the micline of the ganglion, end in a lateral terminus, or leave the ganglion along the ipsilateral cerebral-pleural (C-PI) connective (Fig. 2, $B$ and $D$ ). The lateral terminus is a dense aggregation of fibers just below a small group of neurons in dorsal neuropil (Fig. 2D) and has a curious granular appearance, possibly indicating the presence of numerous synaptic boutons or varicosities. The intensity of labeling of the majority of the small caliber optic fibers decreased rapidly with distance, making it difficult to trace them further. In the best preparations, the small-diameter afferents could be traced about 2 to $2.5 \mathrm{~mm}$.

We carefully examined the area where the optic nerve crosses the cerebral vascular system shortly after it enters the cerebral ganglion (Fig. $3 A$ ). A previous study in this laboratory (Luborsky, 1975) described a close association of the optic nerve and the major vascular vessel of the ganglion (Fig. $3 A$ ), and the limited filling with cobalt in that study suggested that a majority of optic fibers terminated there, possibly to form a neurohemal structure (Luborsky, 1975). Our results from examination of $5-\mu \mathrm{m}$ serial cross-sections showed that optic nerve fibers do not contact this vascular space. Instead, at this point the nerve runs through the sheath just above the cerebral ganglion proper and bccomcs surrounded by a cluster of 30 to 40 neurons that form a small ganglion. This entire structure is encased within a connective tissue sheath and is surrounded by the large vascular vessel (Fig. 3B). This neuron cluster may represent an optic ganglion, which has been described as a more discrete structure in a related species, Aplysia puntata (Eales, 1921), and will be referred to subsequently as the optic ganglion. HRP fills show that optic fibers continue as a coherent tract through the core of this structure (see Fig. $3 B$ ) and, therefore, the fibers are not in a position to directly contact the surrounding vascular spaces.

\section{Uitrastructure of optic fibers}

Many HRP-filled fibers within the optic ganglion and throughout the projection within the cerebral ganglion neuropil have a beaded appearance (Fig. $2 E$ ) suggestive of synaptic varicosities. Areas of the optic tract within the optic ganglion, the lateral terminus, and the midline projection were examined in the electron microscope to characterize putative synaptic sites.

Optic ganglion. The optic nerve can be identified as a discrete structure composed of small-diameter fibers (see Fig. $3 \mathrm{C}$ ) within the core of the optic ganglion. HRP-filled fibers were found only within the optic nerve and never penetrated to the cell body rind of the ganglion or made contact with the sheath or vascular spaces surrounding the optic ganglion. Many fibers in the optic nerve contained only microtubules or occasional mitochondria, but some fibers showed accumulations of small ( 40 to $70 \mathrm{~nm})$, electron-lucent synaptic vesicles and, occasionally, a presumptive synaptic active zone was encountercd (Fig. 3D, asterisk). Much larger axons surround the small-diameter optic tract fibers and delineate the border of the optic nerve and the surrounding optic ganglion (Fig. $3 \mathrm{C}$ ). These large axons typically lack vesicles and did not fill with HRP. Presumably these axons arise from optic ganglion neurons, which form the rind of this structure (Fig. $3 B$ ).

Optic tract midline projection and lateral terminus. HRP-filled fibers in the cerebral ganglion midline projection (see Fig. $2 B$ ) were commonly filled with small $(40$ to $70 \mathrm{~nm}$ ) electron-lucent vesicles and, occasionally, a few scattered small, dense-core vesicles. Vesicles were typically uniformly scattered throughout the cytoplasm of the profile, and defined synaptic active zones were seldom encountered.

The lateral terminus forms a glomerulus-like structure composed of small-diameter fibers which is easily differentiated from the large 

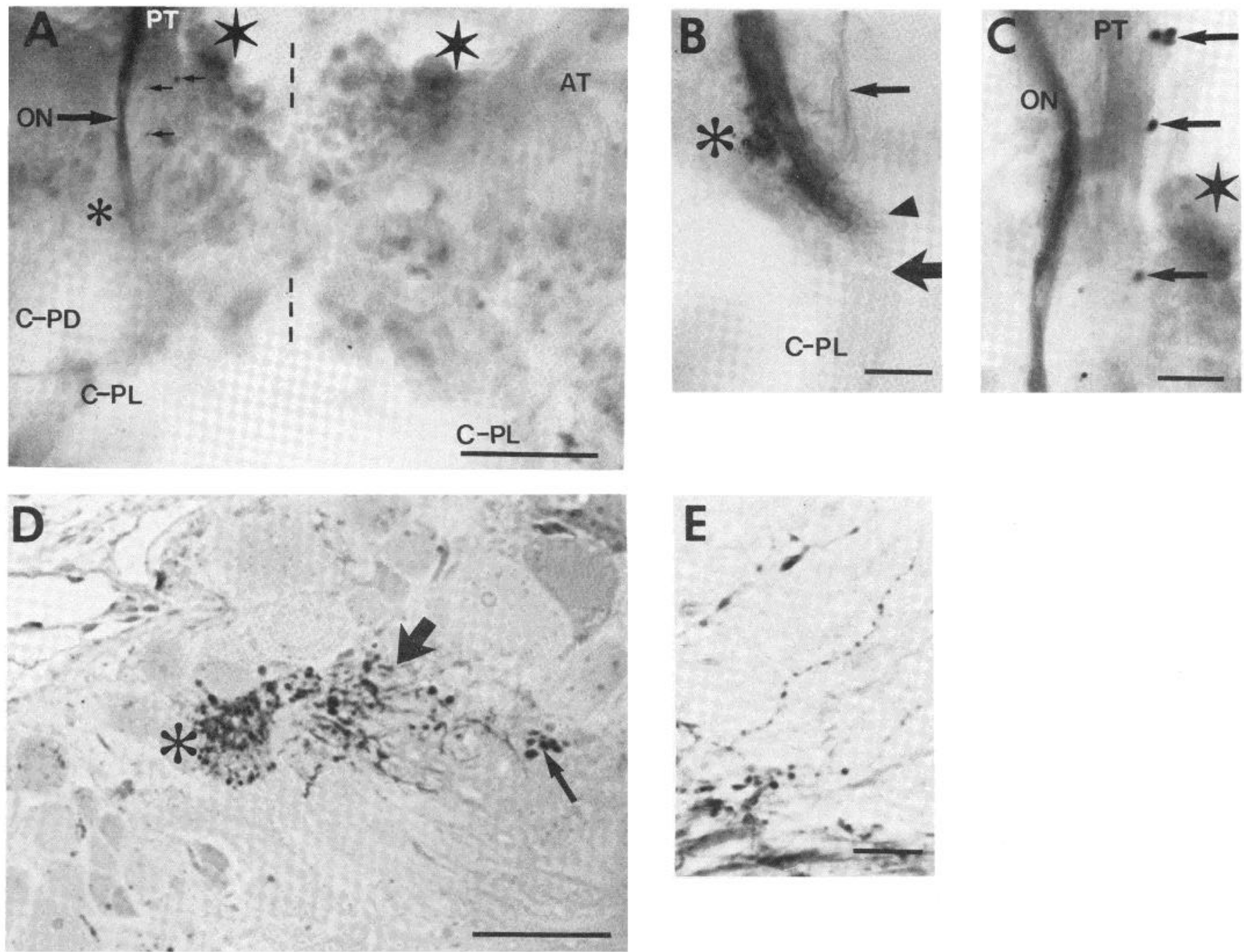

Figure 2. Transport of HRP to the cerebral ganglion from the left optic nerve. A, Dorsal view of a whole mount preparation of the bilaterally symmetrical cerebral ganglion. The stars mark the location of the serotonergic MCGs. The dashed line indicates the midline of the ganglion. The stained optic nerve $(O N$, large arrow) is seen entering the ipsilateral hemiganglion dorsally and rostrally to form the optic tract parallel and lateral to the posterior tentacle (PT) nerve. A small number of stained cell bodies appear along the PT nerve (small arrows). The optic tract extends to caudal neuropil where it divides into three main tracts (see B): a caudal tract, a medial tract, and a lateral bundle (asterisk) that terminates locally. In this preparation, the majority of optic fibers appeared filled. $C-P D$, cerebral-pedal connective; $C-P L$, cerebral-pleural connective. Bar $=600 \mu \mathrm{m}$. B. Higher power micrograph of the optic tract shown in A. This photograph of caudal neuropil was taken after removing the overlying connective tissue sheath. A large group of small caliber fibers (thick arrow) comprises the caudal tract and extends along the C-PI connective. Another bundle of fibers forms a medial trajectory toward the midline of the ganglion (arrowhead) but can be followed for only a short distance. The lateral bundle termiates (asterisk) and has a distinctly granular appearance. A small group of large-diameter fibers (thin arrow) is composed of axons from the neuronal cell bodies in rostral ganglion that are located along the PT nerve ( $A$ and $C$ ). $B a r=80 \mu \mathrm{m}$. C. Micrograph of the optic nerve $(O N)$ and adjacent PT nerve seen in $A$, showing stained efferent neuron cell bodies. The star marks the position of the MCG. Bar = $150 \mu \mathrm{m}$. D. Micrograph of a cross-section (5 $\mu \mathrm{m}$, epoxy embedded) through caudal neuropil at the level of the lateral bundle (asterisk in $A$ and $B$ ) showing the division of the optic tract and the bouton-like strucutres of the terminating lateral tract (asterisk). The main optic tract (thick arrow) continues caudally, but many fibers begin to disperse toward medial neuropil. Larger-diameter axons (thin arrow) are from efferent neurons. $B a r=70 \mu \mathrm{m}$. E , Many small caliber HRP-filled fibers in neuropil have a beaded appearance. Bar $=15 \mu \mathrm{m}$.

axons devoid of vesicles that make up the surrounding neuropil (Fig. $4 A$ ). In contrast to the midline projection, the lateral terminus contained numerous profiles in which vesicles were aligned along a membrane, suggesting the presence of synaptic active zones (Fig. $4 B$ ). HRP-filled profiles commonly formed the presynaptic element, and the postsynaptic element was usually unfilled.

\section{Selective labeling of afferent pathways: $\left[{ }^{3} \mathrm{H}\right]$ Leucine tracing experiments}

Transport of radioactive macromolecules from the eye to the brain dramatically increased the visible extent of the optic nerve projections over that seen in HRP studies. Since incorporation of amino acids into transportable macromolecules occurs only at the soma (see Hendrickson and Edwards, 1978), this method labels only the optic afferents. Exposure of the eye to $\left[{ }^{3} \mathrm{H}\right]$ leucine equally labels both photoreceptors and neurons, so that the axons of both of these types should be labeled. As with HRP, no differences were seen in projections from the right or left eye.

The radiolabeled optic nerve enters the cerebral hemiganglion rostrally, passes through the optic ganglion, and continues to caudal neuropil as a distinct fiber tract (Fig. $5 A$ ). Label was confined to the core of the optic ganglion and did not approach the surrounding vascular spaces, consistent with results of HRP tracing. In caudal ganglion a lateral branch leaves the main tract and terminates (Fig. 

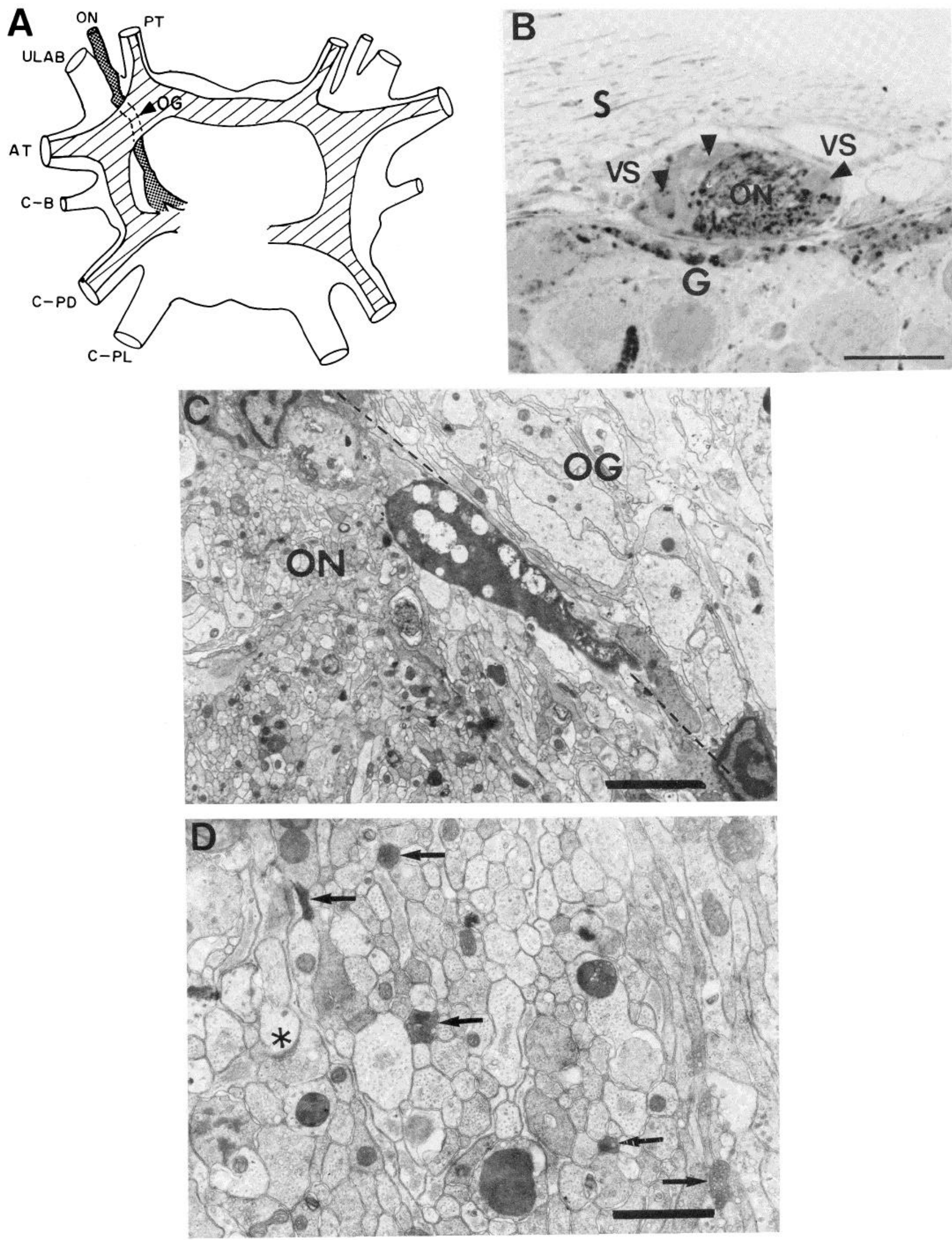

Figure 3 


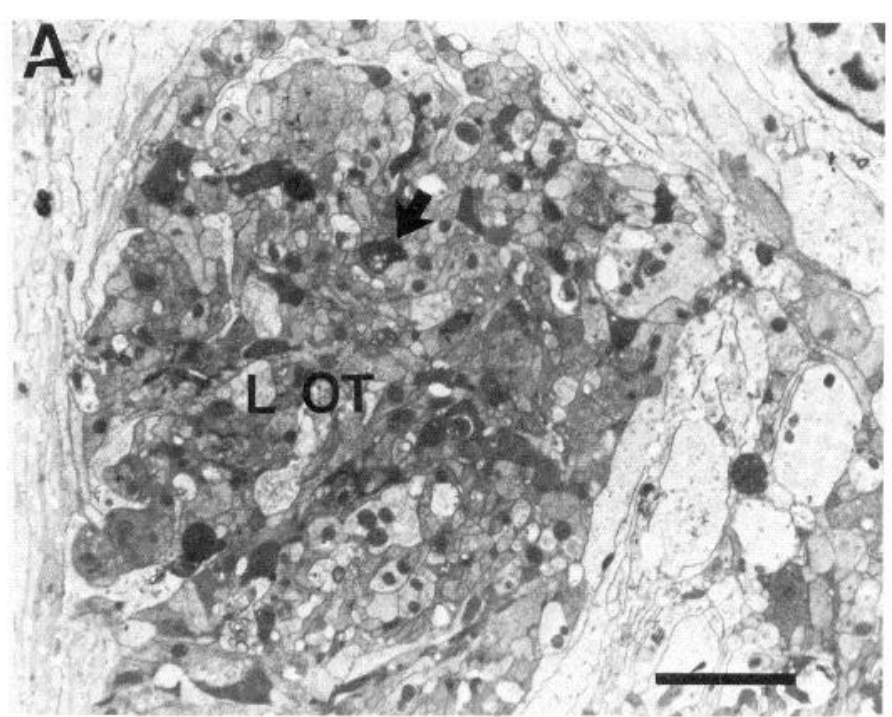

$5 E)$, as in HRP tracing studies. However, the $\left[{ }^{3} \mathrm{H}\right]$ leucine-labeled projection within the cerebral ganglion was much more extensive than was seen with HRP. The midline optic tract projection is massive (Fig. $5 C$ ), with a major tract extending to the contralateral cerebral neuropil (Fig. 5, $C$ and $D$ ). Radiolabeled fibers extend beyond the ganglion along several routes in addition to the ipsilateral C-PI projection seen with HRP; large tracts project along the contralateral as well as the ipsilateral C-PI connective (Fig. 5, $C$ and D), and along the ipsi- and contralateral cerebral-pedal (C-Pd) connectives. These projections extend to the paired pleural and pedal ganglia, respectively. Smaller projections leave the cerebral ganglion along the paired PT, anterior tentacle (AT), and upper lip (ULAB) nerves. Part of the pleural projections (Fig. $6 A$ ) continues beyond these ganglia along the PI-Ab connectives (Fig. $6 B$ ) that connect to the abdominal ganglion. Labeled tracts could be followed easily some distance along the PI-Ab connectives that remained attached to the pleural ganglia (see Fig. $6 B$ ), which is about 3 to $3.5 \mathrm{~cm}$ from the labeled eye, but they could not be detected either within the abdominal ganglion or within the millimeter or so of connective attached to that ganglion. The large portion of intervening connective (up to $5 \mathrm{~cm}$ ) was not examined in detail; thus, we are unable to determine the

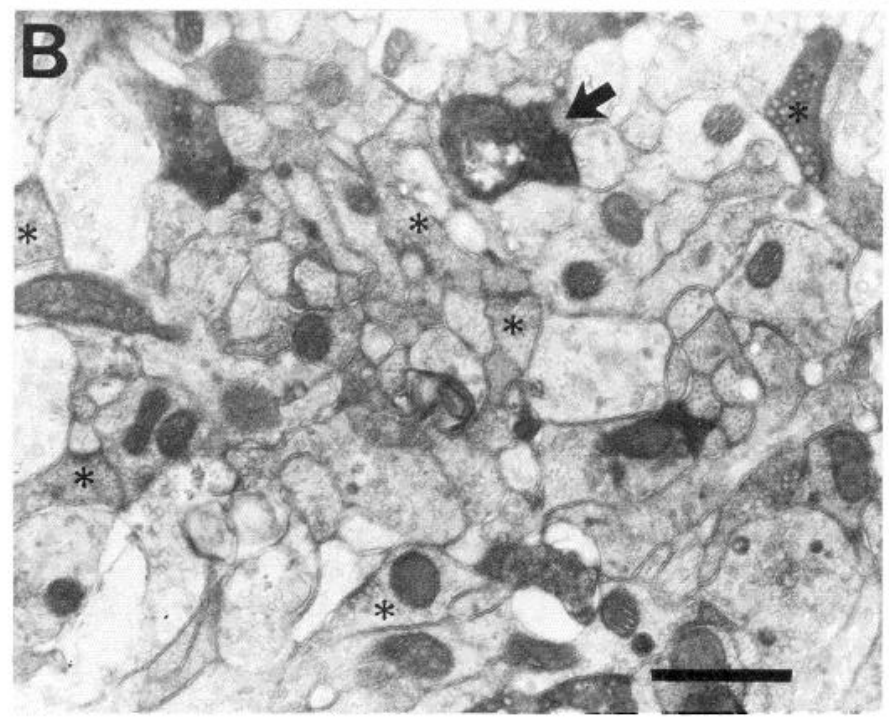
total distance of $\left[{ }^{3} \mathrm{H}\right]$ leucine transport. Projections to pedal ganglia terminated within pedal neuropil.

In most preparations, the contralateral ganglionic projections, although substantial, appeared somewhat smaller than ipsilateral projections. Throughout the CNS projections, labeled fibers are predominantly within definite tracts within neuropil, although a few individual, varicose fibers were occasionally seen to penetrate to the cell body layer. CNS neuronal somata never labeled, supporting the idea that leucine-labeled material is transported only in the orthograde direction. A summary diagram of the optic nerve $\left[{ }^{3} \mathrm{H}\right]$ leucinelabeled afferent projection from the eye throughout the cerebral ganglion is shown in Figure $5 F$.

\section{Selective labeling of pacemaker projections}

When eyes were exposed to low concentrations of $\left.{ }^{3} \mathrm{H}\right] \mathrm{DOPA}$, $\left[{ }^{3} \mathrm{H}\right] \mathrm{DA}$, or $\left[{ }^{3} \mathrm{H}\right]-5$ - HTP $\left(2\right.$ to $\left.4 \times 10^{-7} \mathrm{M}\right)$, label concentrated over neuronal cell bodies in the outer retinal layer which have been previously identified (Jacklet et al., 1982) as pacemaker neurons (Fig. 7A). These neurons may be dopaminergic, as suggested by induced fluorescence studies (Luborsky-Moore and Jacklet, 1976b). No specific labeling of any neural structures was seen with $\left[{ }^{3} \mathrm{H}\right] \mathrm{NE}$, consistent with other findings that Aplysia CNS contains little or no NE (Carpenter et al., 1971). 5-Hydroxytryptamine itself was not tested in this study, as other work (Turner and Cottrell, 1978; L. M. Olson and J. W. Jacklet, unpublished observation) suggests that it is specifically taken up by serotonergic terminals, but not cell bodies. Photoreceptors, located in the inner retinal layer, did not accumulate any of the labeled compounds, even at higher concentrations ( 2 to $4 \times 10^{-6} \mathrm{M}$ ) that produced nonspecific labeling of the sheath and non-neural elements. $\left[{ }^{3} \mathrm{H}\right] \mathrm{DOPA}$ provided the most pronounced labeling of pacemaker neurons.

Figure 4. Electron micrographs of HPR-filled fibers in the lateral terminus 列 of the optic tract ( $L O T$ ) forms a discrete structure within neuropil because the small fibers of this bundle are surrounded by neuropil that consists of large-diameter fibers typically devoid of vesicles. Bar $=2.6 \mu \mathrm{m}$. $B$, Higher power micrograph of an area in $A$ (match large arrows). Numerous presumptive synaptic active zones, with vesicles aligned along the presynaptic membrane, occur in this area (asterisks). The postsynaptic elements were not usually stained with HRP and often lacked vesicles. There were many more active zones here than in other parts of the optic projection. Bar $=0.8$ $\mu \mathrm{m}$.

Figure 3. $A$ and $B$, Association of the optic nerve with vascular spaces of the cerebral ganglion. $A$, Sketch of the cerebral ganglion, showing the major blood vessel (diagonally shaded area) that infilitrates the sheath of the ganglion. The optic nerve $(O N)$ passes beneath this vessel and through the optic ganglion (OG) as it enters the cerebral ganglion (arrowhead). (Adapted from Luborsky, 1975). B, In cross-sections through this area, the HPR-filled optic nerve $(O N)$ travels in the sheath $(S)$ above the cerebral ganglion proper $(G)$. It passes through a cluster of neurons (arrowheads) which form the optic ganglion. The optic ganglion is surrounded by vascular spaces (VS). The dark structures in the cerebral ganglion just beneath the optic ganglion are pigmented inclusions in neurons that in the microscope are easily discriminated from HRP. HRP-filled fibers were seen only in the optic nerve within the core of the optic ganglion. Bar $=60 \mu \mathrm{m} . C$ and $D$. Electron micrographs of the optic nerve and optic ganglion. $C$, the optic nerve $(O N)$ is made up of small fibers $(0.2$ to $0.6 \mu \mathrm{m})$ and is easily differentiated from the surrounding optic ganglion (OG) axons, which are much larger. The neuronal cell bodies of the optic ganglion (not shown) occupy a more peripheral position. The large dark, club-like profile at the border of the optic nerve and ganglion is probably an efferent axon filled with HRP. The dashed line demarcates the boundary between the optic nerve and the optic ganglion. Bar $=3.4 \mu \mathrm{m}$. $D$, Some optic nerve axons within the optic ganglion show accumulations of small electron-lucent vesicles, but presumptive synaptic profiles were not frequently encountered. One possible synaptic active zone is shown in this micrograph (asterisk), but neither profile contains HRP. This preparation contained relatively few HRP-stained fibers, which are indicated by the arrows. Bar $=1.2 \mu \mathrm{m}$. 

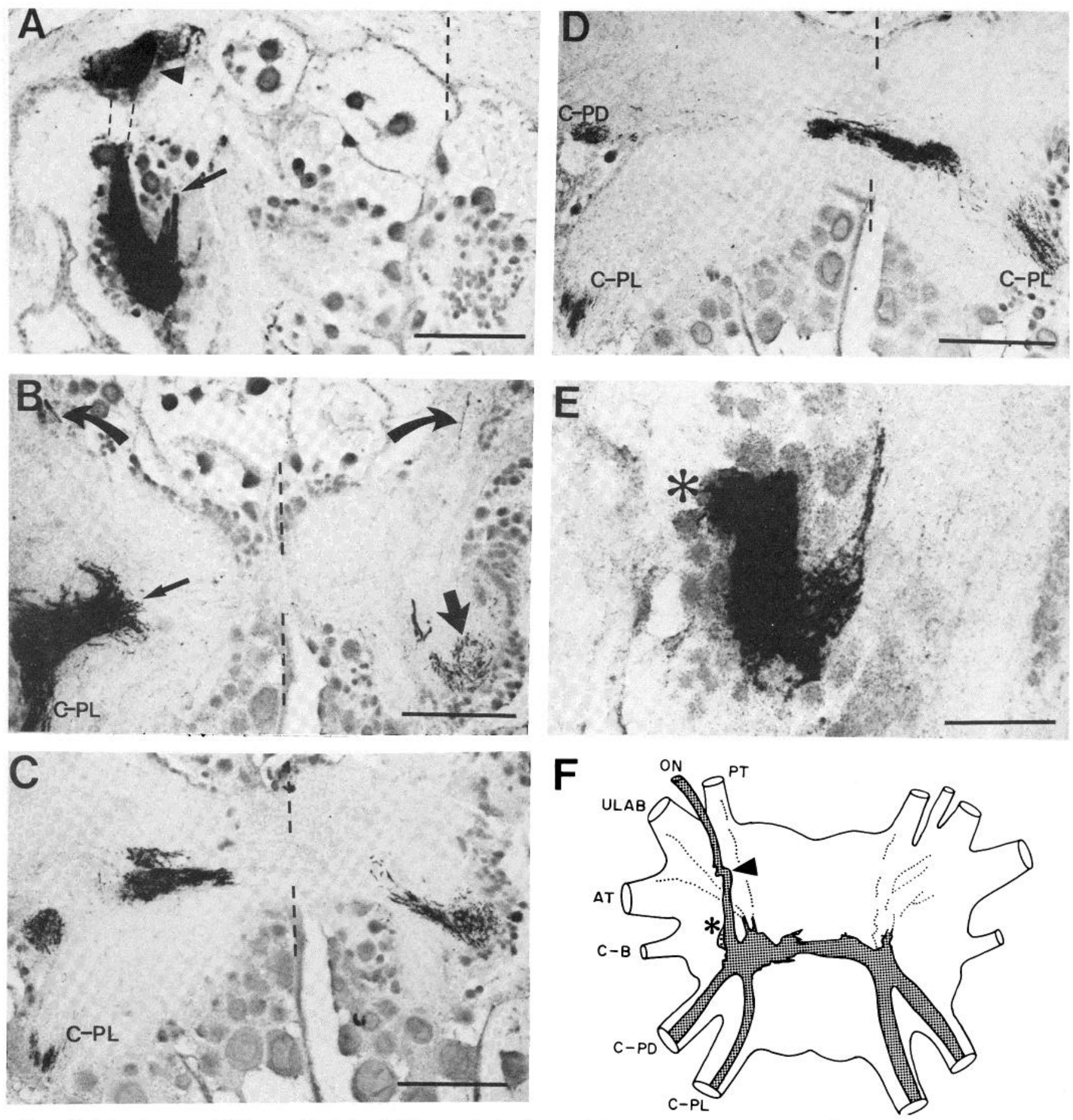

Figure 5. Autoradiograms of $\left[{ }^{3} \mathrm{H}\right]$ leucine-labeled material transported to the cerebral ganglion in the left optic nerve' (horizontal $12-\mu \mathrm{m}$ paraffin sections counterstained with azure-eosin). Eyes were incubated in labeled medium for $2 \mathrm{hr}$ and the preparation was maintained for an additional $20 \mathrm{hr}$ in unlabeled medium for transport of labeled material. $A$ to $D$ are sections from a dorsal-to-ventral sequence. In all micrographs, the top is rostral and the midline of the ganglion is indicated by the dashed line. $A$, The labeled left optic nerve enters the ganglion dorsally, becomes associated with the optic ganglion (arrowhead), and forms an optic tract that extends to caudal neuropil. The beginning of an anterior projection (arrow) can be seen at this level, which will form projections along three anterior cerebral nerves. $B$, Ventral to $A$, a massive projection is seen leaving the ganglion along the C-PI connective. The medial projection is extensive (small arrow) and a projection in contralateral neuropil (large arrow) is apparent. Rostrally, small projections extend bilaterally along the paired PT nerves (curved arrows). $C$ and $D$, The optic tract crosses the midline at this level to form the contralateral projection. The projection along the contralateral C-PI connective and the ipsilateral C-PI and C-Pd connectives are also evident. Bars $(A$ to $D)=260 \mu \mathrm{m}$. $E$, As seen in HRP studies, the optic tract forms a distinctive lateral bundle terminating in caudal neuropil (asterisk). Bar $=100 \mu \mathrm{m}$. F, Summary diagram of optic afferent projections in the cerebral ganglion. Optic afferents from a single optic nerve $(O N)$ enter the sheath of the cerebral ganglion, pass through the optic ganglion at the arrowhead, and project to the ipsi- and contralateral ganglion. Small projections (dotted lines) extend anteriorly along the paired At, PT, and ULAB nerves. Major tracts leave the ganglion along the bilateral C.Pl and C-Pd connectives. The asterisk identifies the terminal lateral bundle seen in $E$. 

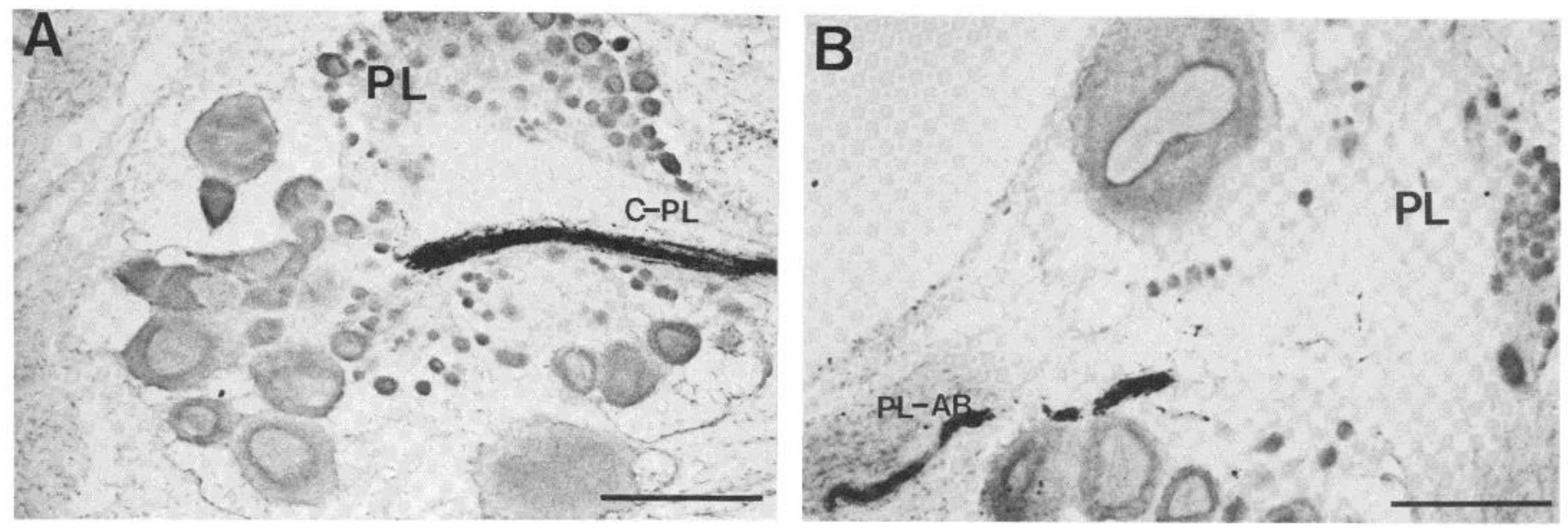

Figure 6. $\left[{ }^{3} \mathrm{H}\right]$ Leucine autoradiograms showing optic tract projections to pleural ganglia $(P L)$ (horizontal $12-\mu \mathrm{m}$ paraffin sections, counterstained with azure-eosin). Rostral is to the right in the micrographs. A, A large group of fibers enters the pleural ganglion along the C-PI connective. $B$, Some of these fibers leave the ganglion along the PI-Ab connective to the abdominal ganglion. The large neuron at the top of the micrograph is the left pleural giant cell. $B a r=260 \mu \mathrm{m}$.

To test the specificity of labeling of aminergic compounds, the cerebral ganglion, which contains the identified, paired serotonergic metacerebral giant neurons (MCGs), was exposed to these compounds. Labeling with $\left[{ }^{3} \mathrm{H}\right]-5-\mathrm{HTP}\left(2 \times 10^{-7}\right.$ to $\left.2 \times 10^{-6} \mathrm{M}\right)$ is restricted to the MCGs and a small subset of neurons located primarily in rostral ganglion surrounding the MCGs. $\left[{ }^{3} \mathrm{H}\right] \mathrm{DA}$ and $\left[{ }^{3} \mathrm{H}\right]$ DOPA also lightly label the MCGs, but much more intense accumulation is seen with $\left[{ }^{3} \mathrm{H}\right]-5-\mathrm{HTP}$. All compounds labeled only a restricted subset of CNS neurons.

After eyes were exposed to $\left[{ }^{3} \mathrm{H}\right] \mathrm{DA},\left[{ }^{3} \mathrm{H}\right] \mathrm{DOPA}$, and $\left[{ }^{3} \mathrm{H}\right]-5-\mathrm{HTP}$, and time was allowed for tritium-incorporated material to transport to the attached ganglia, $\left[{ }^{3} \mathrm{H}\right] \mathrm{DOPA}$ - and $\left[{ }^{3} \mathrm{H}\right] \mathrm{DA}$-labeled tracts, but no $\left[{ }^{3} \mathrm{H}\right]-5$-HTP-labeled tracts, were seen within the brain (Fig. $7 B$ ). With prolonged exposure of eyes to the labeled medium during transport experiments $(24 \mathrm{hr})$, more background labeling, including label over photoreceptors, occurred. However, levels over photoreceptors did not approach that seen over pacemaker neurons and, thus, was taken to represent nonspecific uptake as it was comparable to that seen over non-neural structures of the eye. $\left[{ }^{3} \mathrm{H}\right] \mathrm{DOPA}$ labeling of eyes produced the clearest CNS tracts, probably due to the higher intensity of labeling at the cell bodies. Labeled projections within the cerebral ganglion were bilateral, with an extensive distribution throughout caudal neuropil. In the most heavily labeled preparations (2 of 8 ), projections could also be followed out of the cerebral ganglion along the paired C-PI and C-Pd connectives to the pleural and pedal ganglia, and beyond the pleural ganglia along the $\mathrm{PI}-\mathrm{Ab}$ connectives, distances totaling up to $3 \mathrm{~cm}$, from the eye to the PI-Ab connective leaving the pleural ganglion. Because $\left[{ }^{3} \mathrm{H}\right]$ DOPA-labeled tracts were much lighter than $\left[{ }^{3} \mathrm{H}\right]$ leucine-labeled tracts (see Fig. $7 B$ ), it was difficult to determine whether the small projections along the anterior cerebral nerves that were seen with $\left[{ }^{3} \mathrm{H}\right]$ leucine were present. However, a projection along the ipsilateral PT nerve could be detected. The reconstruction of the $\left[{ }^{3} \mathrm{H}\right] \mathrm{DOPA}$ labeled optic projection within the cerebral ganglion (Fig. $7 D$ ) is very similar to that of the $\left[{ }^{3} \mathrm{H}\right]$ leucine projection (cf. Figs. $5 F$ and $7 D$ ).

In about half of the first few (2 of 4$)\left[{ }^{3} \mathrm{H}\right] \mathrm{DOPA}$ transport experiments, a small number of labeled cell bodies were detected in the ipsilateral rostral cerebral ganglion (Fig. 7C), although this had never been seen with $\left[{ }^{3} \mathrm{H}\right]$ leucine transport. Additionally, labeled axons of a size and distribution unlike the afferent projections of $\left[{ }^{3} \mathrm{H}\right]$ leucine were seen within the ipsilateral PT nerve. The possibility of a leak between the chambers of the incubation dish, which would directly expose the ganglion to the labeled medium, was eliminated because nonspecific background labeling of the ganglion was extremely low and, more importantly, only structures ipsilateral to the labeled eye were labeled. It seemed most likely that these cerebral ganglion cell bodies became labeled by retrograde transport of $\left[{ }^{3} \mathrm{H}\right] \mathrm{DOPA}$ after uptake into nerve terminals in the eye. However, the $\left[{ }^{3} \mathrm{H}\right] \mathrm{DOPA}$ labeled cerebral ganglion neurons were not the same as those previously identified in HRP backfills, which are located more laterally along the PT nerve. Those neurons never became labeled. This suggested that axons from the $\left[{ }^{3} \mathrm{H}\right] \mathrm{DOPA}$-labeled cerebral neurons do not project to the eye through the optic nerve, but through some alternate route. Further exploration confirmed that these fibers reach the eye through an accessory optic nerve (Olson and Jacklet, 1984). A manuscript on this is being prepared.

\section{Electrophysiological confirmation of pacemaker projections to central ganglia}

The synchronous firing of pacemaker neurons results in a distinctive CAP that is easily recorded from the optic nerve (Jacklet et al., 1982). The $\left[{ }^{3} \mathrm{H}\right]$ leucine transport experiments clearly identified optic afferents in the C-PI, C-Pd, and PI-Ab connectives and the anterior nerves of the cerebral ganglion. [ $\left.{ }^{3} \mathrm{H}\right] \mathrm{DOPA}$ studies indicated that at least some of these afferents were from the circadian pacemaker neurons in the retina. If the $\left[{ }^{3} \mathrm{H}\right] \mathrm{DOPA}$-labeled fibers are axons of pacemaker neurons, it should be possible to record the pacemaker $\mathrm{CAP}$ in these pathways as well.

Recording simultaneously from connectives and the optic nerve revealed a small, slow, triphasic potential that is strictly time-locked to the larger, spontaneously occurring optic nerve CAP, in the ipsilateral C-PI and C-Pd connectives and the contralateral C-PI connective (Fig. 8, $A$ to $C$ ). These connective potentials have the same waveform and time course as the distinctive, long-duration optic nerve CAP, suggesting that the recording is from pacemaker axons. A similar potential was not seen in the PI-Ab connective, possibly due to fewer contributing fibers and an unfavorable signalto-noise ratio.

A slow, triphasic antidromic potential was recorded in the optic nerve after electrical stimulation of C-PI and C-Pd connectives, confirming that optic axons extend to these connectives (Fig. $8, D$ and $E$ ). The antidromically activated potential has a waveform and time course very similar to those of the orthodromic CAP seen in connective recordings and the spontaneous CAP in the optic nerve that originates in the eye (Fig. $8 D$ ). Stimulation of the PI-Ab connective also evoked an optic nerve potential very similar to that seen with C-PI and C-Pd stimulation (Fig. 8E), whereas stimulation of other pleural nerves did not.

In contrast to the slow antidromic optic nerve potential seen with connective stimulation, stimulation of ipsilateral cerebral AT and PT 

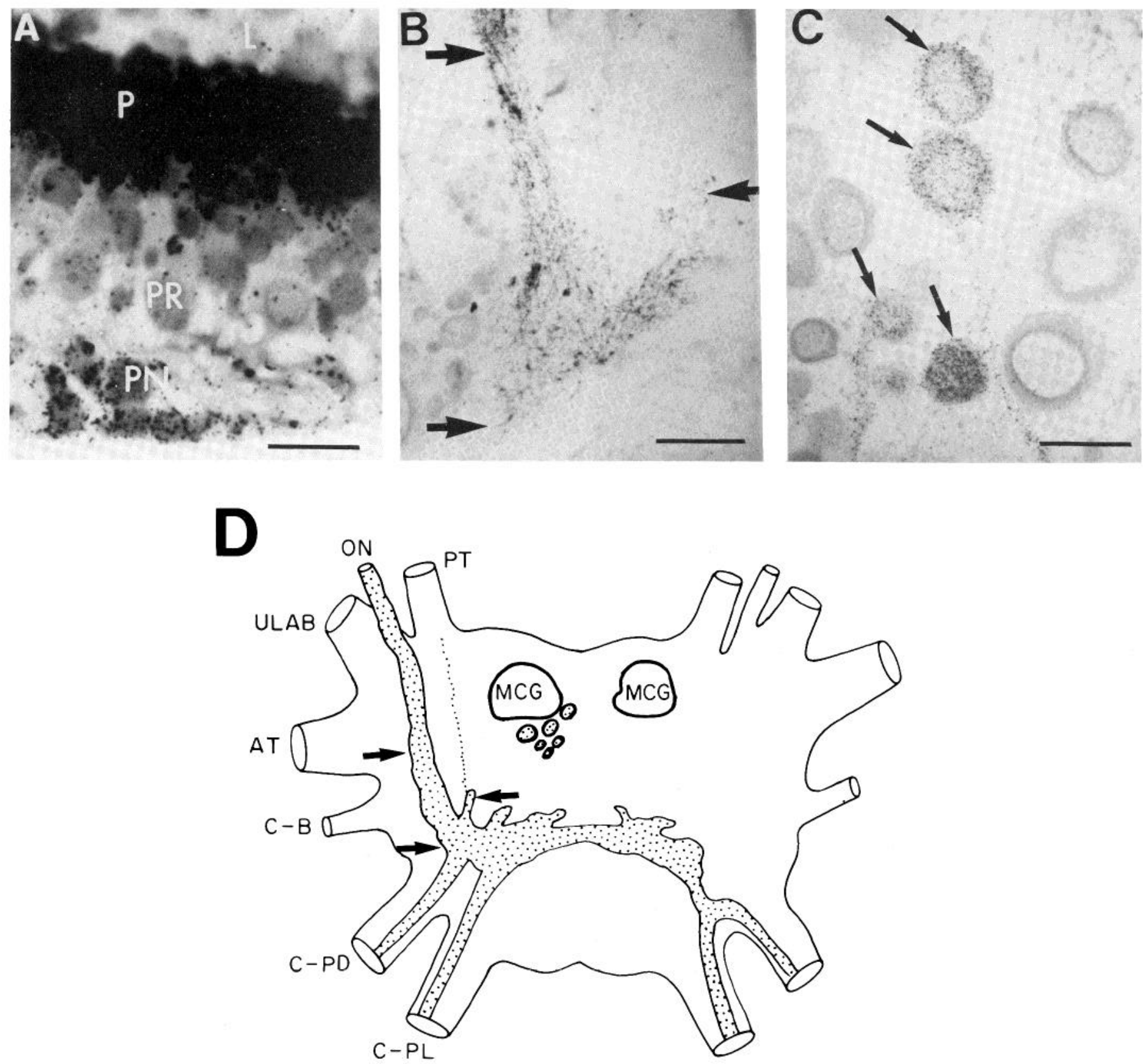

Figure 7. Autoradiograms showing selective labeling of retinal neurons with $\left[{ }^{3} \mathrm{H}\right] \mathrm{DOPA}$, and $\left[{ }^{3} \mathrm{H}\right] \mathrm{DOPA}$ cerebral ganglion projections. $A$, Labeling of the eye with $2.5 \times 10^{-7} \mathrm{M}\left[{ }^{3} \mathrm{H}\right] \mathrm{DOPA}$ for $8 \mathrm{hr}$ results in accumulation of label over a cluster of pacemaker neurons $(P N)$ in the outer retinal layer. Photoreceptors $(P R)$, which make up the inner retinal layer, do not accumulate label. The unlabeled dark pigmented band $(P)$ between the lens $(L)$ and the photoreceptor layer is composed of pigmented support cells interspersed with distal segments of the photoreceptors (5- $\mu \mathrm{m}$ epoxy section, counterstained with azure II/ methylene blue). $B a r=30 \mu \mathrm{m} . \mathrm{B},\left[{ }^{3} \mathrm{H}\right] \mathrm{DOPA}$-labeled tracts within the cerebral ganglion. Although this projection is much lighter than that labeled with $\left.{ }^{3} \mathrm{H}\right]$ leucine, it is very similar. The arrows are for reference to the reconstruction in $D$. Bar $=80 \mu \mathrm{m}$. C, A small number of neurons in ipsilateral anterior cerebral ganglion were also labeled. These neurons were probably labeled by retrograde transport of the $\left[{ }^{3} \mathrm{H}\right] \mathrm{DOPA}$. ( $B$ and $C$ are $12-\mu \mathrm{m}$ horizontal paraffin sections, counterstained with azure-eosin). Bar $=55 \mu \mathrm{m}$. D. Reconstruction of the $\left[{ }^{3} \mathrm{H}\right] \mathrm{DOPA}$ optic tract projection in the cerebral ganglion. The arrows correspond to the arrows shown in $B$. No obvious differences between $\left[{ }^{3} \mathrm{H}\right] \mathrm{DOPA}$ and $\left[{ }^{3} \mathrm{H}\right]$ leucine projections were seen (compare with Fig. $5 F$ ), although the small projections along most anterior cerebral nerves were difficult to resolve with $\left[{ }^{3} \mathrm{H}\right] \mathrm{DOPA}$, and $\left[{ }^{3} \mathrm{H}\right] \mathrm{DOPA}$ labeled some cerebral neurons (strippled circles below the ipsilateral MCG), presumably by retrograde transport. However, the positions of $\left[{ }^{3} \mathrm{H}\right] \mathrm{DOPA}$-labeled neurons do not overlap with efferent neurons stained with HRP (see Fig. $1 A$ ).

nerves evoked a volley of short-duration unitary potentials, many of which fatigued rapidly with repeated stimulation. With PT stimulation, some potentials did not fatigue with high frequency stimulation. These potentials were also of short duration and did not resemble the CAP seen with connective stimulation. However, by superimposing traces during repeated stimulation of the PT nerve, a small CAP. like potential underlying the nonfatiguing unitary potentials (not shown) was revealed. No CAPs were recorded in other anterior nerves.

\section{Discussion}

These experiments demonstrate that optic fibers are distributed throughout virtually the entire Aplysia CNS. HRP and $\left[{ }^{3} \mathrm{H}\right]$ leucine tracing provide evidence that optic fibers project into the neuropil of 

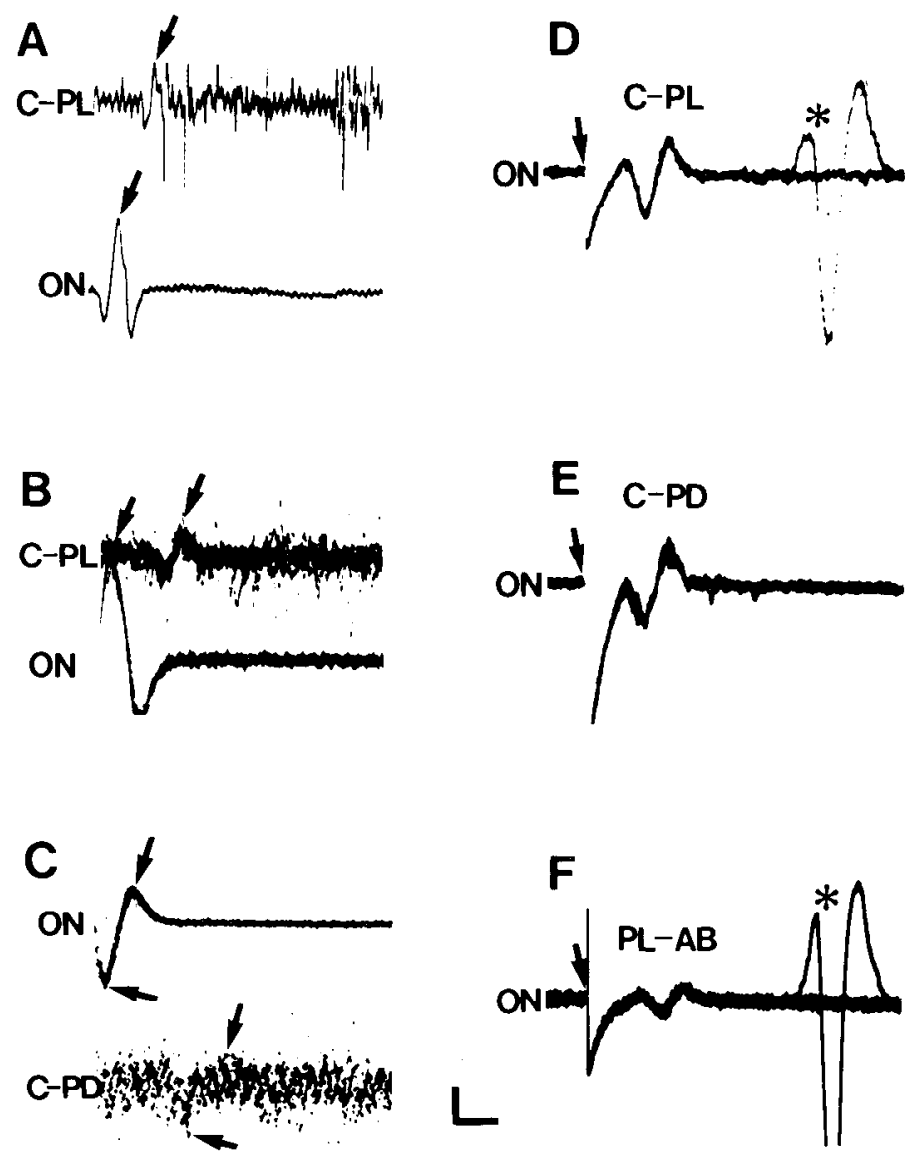

Figure 8. Recordings of electrical activity trom the optic nerve and central connectives. $A$ to $C$, Simultaneous recordings of the spontaneous CAP in the optic nerve (ON) and time-locked potentials in C-PI and C-Pd connectives (for comparison, arrows mark peaks and/or troughs of the CAP potential in both connective and optic nerve recordings. $A, A$ single sweep of the oscilloscope shows a small CAP in the C-PI connective that has a waveform and timecourse very similar to those of the distinctive spontaneous optic nerve CAP (arrows). Upper trace, $5 \mu \mathrm{V}$; lower trace, $20 \mu \mathrm{V} ; 100 \mathrm{msec} . B_{\text {, }}$ Superposition of 10 consecutive traces triggered by the spontaneous optic nerve CAP emphasizes the time-locked potential in the C-PI. The trace intensity was turned down to reduce the visibility of potentials not correlated with the optic nerve CAP. Upper trace, $5 \mu \mathrm{V}$; lower trace, $20 \mu \mathrm{V} ; 100 \mathrm{msec}$. $C$, A much smaller time-locked potential was recorded from the C-Pd connective by superimposing numerous (13) traces. Upper trace, $20 \mu \mathrm{V}$; lower trace, $5 \mu \mathrm{V} ; 100 \mathrm{msec}$. $D$ to $F$, Antidromic optic nerve CAPs. Electrical stimulation (arrows) of C-PI $(D)$, C-Pd $(E)$, and $\mathrm{Pl}-\mathrm{Ab}(F)$ connectives evoke a slow triphasic potential in the optic nerve (asterisks). Each record shows five superimposed traces. Bar $=20 \mu \mathrm{V}, 50 \mathrm{msec}$. In $D$, the antidromic potential $(\mathrm{C}-\mathrm{PI})$ is nearly identical in form to the spontaneous CAP (asterisks in $D$ and $F$ ) originating in the eye. The same waveform is evoked by C-Pd stimulation $(E)$, and a reduced but similar potential is evoked by $\mathrm{Pl}-\mathrm{Ab}$ stimulation $(F)$.

the cerebral ganglion, in contrast to earlier cobalt tracing experiments which suggested that the nerve terminated shortly after entering the ganglion (Jahan-Parwar and Fredman, 1976; Luborsky-Moore and Jacklet, 1976a). It was difficult to determine the full extent of the optic nerve projection using HRP due to the limited filling of the optic afferents and the decrease in concentration of the reaction product with distances. $\left[{ }^{3} \mathrm{H}\right]$ Leucine autoradiography confirmed the projection seen with HRP and provided a much more complete visualization of the total projection of optic afferents. The observed tracts represent the combined projections of pacemaker neurons and photoreceptors. Both cell types in the eye were equally labeled with leucine and both are known to contribute axons to the optic nerve (Jacklet and Rolerson, 1982).
The exlensive optic nerve projections revealed by transport of $\left[{ }^{3} \mathrm{H}\right]$ leucine-labeled macromolecules are unlikely to be labeled as a result of transneuronal relay of material because this usually requires massive quantities of the precursor and long survival times (see Jones and Hartman, 1978). In this study, labeled projections seen after $12 \mathrm{hr}$ of transport are essentially unchanged after longer transport times (i.e., up to $36 \mathrm{hr}$ ), which strongly argues that the observed projections represent only the retinal axons themselves. Moreover, electrical recordings of connective potentials correlated with optic nerve CAPs also argues against a polysynaptic pathway.

Although the major optic tracts from each eye labeled with leucine project bilaterally to most rostral ganglia, we were unable to trace the projection directly to the abdominal ganglion, probably due to the extremely long transport distances $(>8 \mathrm{~cm}$ ) and the probable large reduction in the number of fibers contributing to the longer projections.

Selective identification of pacemaker projections using [ $\left.{ }^{3} \mathrm{H}\right]$ DOPA transport. We find that pacemaker neurons, which occupy a characteristic position in the outer retina (Jacklet et al., 1982) will selectively accumulate exogenous $\left[{ }^{3} \mathrm{H}\right] \mathrm{DOPA},\left[{ }^{3} \mathrm{H}\right] \mathrm{DA}$, and $\left[{ }^{3} \mathrm{H}\right]-5$ HTP. Photoreceptors, which are easily distinguished from pacemaker neurons by their distinctive morphology and position in the inner retinal layer (Jacklet and Rolerson, 1982), do not accumulate these compounds. This specific labeling pattern allowed us to selectively trace the axons of the pacemaker neurons.

A high affinity uptake mechanism in aminergic neurons for their transmitter or its precursors has long been established for vertebrates (see lversen, 1975) and has also been described in molluscan aminergic neurons (Pentreath and Cottrell, 1972, 1973; Pentreath and Berry, 1978; Bailey et al., 1983). This mechanism has been widely exploited to label neurons based on their transmitter content (Aghajanian and Bloom, 1967). DA may be the transmitter of the Aplysia pacemaker neurons because it is found in the eye (Corrent et al., 1978) and is synthesized there (Loh and Jacklet, 1977). Pacemakers contain dense-core vesicles that react positively in the Wood's reaction for detection of catecholamines (Luborsky-Moore and Jacklet, 1977), and have a formaldehyde-induced fluorescence characteristic of catecholamines (Luborsky-Moore and Jacklet, 1976b). Because NE has not been detected in Aplysia (Carpenter et al., 1971), it is thought that the catecholamine detected by these reactions is DA.

However, selective uptake of $\left[{ }^{3} \mathrm{H}\right] \mathrm{DOPA}$ and $\left[{ }^{3} \mathrm{H}\right] \mathrm{DA}$ by these neurons cannot be used to positively identify the pacemaker transrriller, since they also accumulate low levels of the precursor to serotonin, $\left[{ }^{3} \mathrm{H}\right]-5-\mathrm{HTP}$. Studies in vertebrates have indicated that aminergic neurons may have some affinity to take up other aminergic transmitters (Lichtensteiger et al., 1967; Shaskan and Snyder, 1970; Berger and Glowinski, 1978). Also, in molluscs, Turner and Cottrell (1978) report that both serotonergic and dopaminergic neurons in Planorbis equally accumulated DA, DOPA, and 5-HTP. Uptake was restricted, however, to a small subset of CNS neurons, possibly all utilizing related amines as transmitters. We obtained similar results using the identified serotonergic MCGs to examine the specificity of uptake in Aplysia. The MCGs and an additional small subset of cerebral neurons accumulate $\left[{ }^{3} \mathrm{H}\right]-5-\mathrm{HTP}$, but they also accumulate smaller quantities of $\left[{ }^{3} \mathrm{H}\right] \mathrm{DOPA}$ and $\left[{ }^{3} \mathrm{H}\right] \mathrm{DA}$. [ $\left.{ }^{3} \mathrm{H}\right]-5-\mathrm{HTP}$ labels a population of neurons in the cerebral ganglion that has the same location as serotonergic neurons identified by serotonin antibody staining (Goldstcin et al., 1984; Ono and McCamen, 1984) and glyoxylic acid-induced fluorescence (Tritt et al., 1983; L. M. Olson and $\mathrm{J}$. W. Jacklet, unpublished observations). Ocular pacemaker neurons and the small group of CNS neurons just described are the only neurons that selectively accumulate the tested aminergic transmitters or precursors when these compounds are available to nervous tissue in low concentrations $\left(2 \times 10^{-7} \mathrm{M}\right)$. This selective labeling suggests that pacemaker neurons possess a high affinity uptake system for these compounds, and supports the idea that they utilize an aminergic transmitter, possibly DA. 
Exposure of eyes to $\left[{ }^{3} \mathrm{H}\right] \mathrm{DOPA}$ results in a pattern of labeled tracts in the CNS very similar to that seen in $\left[{ }^{3} \mathrm{H}\right]$ leucine tracing experiments. Projections are bilateral to cerebral, pleural, and pedal ganglia, and they leave the pleural ganglia along the PI-Ab connective. It is likely that these tracts represent the fast transport of DA, synthesized in the cell body from selectively accumulated [ $\left.{ }^{3} \mathrm{H}\right] \mathrm{DOPA}$ and packaged into storage vesicles (Goldberg and Schwartz, 1980). The ability of a transmitter to be accumulated into vesicles and, subsequently, fast transported, may depend on its chemical similarity to the neuron's native transmitter (for reviews see Schwartz, 1979; Grafstein and Forman, 1980), again suggesting that pacemaker neurons may be aminergic. It is not clear why we were unable to detect labeled tracts within the CNS after exposure of the eye to $\left[{ }^{3} \mathrm{H}\right]-5-\mathrm{HTP}$, which specifically labels pacemaker neurons, since other studies suggest that uptake into storage vesicles, and subsequent transport, does not distinguish between aminergic compounds (see Schwartz, 1979). The amount of [ $\left.{ }^{3} \mathrm{H}\right]-5$-HTP label over pacemaker neurons was much less than that seen with $\left[{ }^{3} \mathrm{H}\right] \mathrm{DOPA}$, however (also, note differences in specific activity of these compounds; see "Materials and Methods"), and transported label may have been too light to be resolved.

The low level of label over photoreceptors compared to pacemaker neurons makes it unlikely that they contribute to the described projections. Furthermore, evidence that non-aminergic neurons may not be able to transport aminergic transmitters (Goldman and Schwartz, 1974) suggests that photoreceptors, which do not appear to contain amines (Luborsky-Moore and Jacklet, 1976b, 1977), would not be able to transport any nonspecifically incorporated $\left[{ }^{3} \mathrm{H}\right]$ UOPA that might occur during prolonged exposure to the labeled medium.

Pacemaker activity in CNS pathways. The results from $\left[{ }^{3} \mathrm{H}\right] \mathrm{DOPA}$ tracing studies showing pacemaker axons extending to pleural, pedal, and possibly abdominal ganglia, were corroborated by records of a CAP in connectives leaving the cerebral ganglion that were correlated with the spontaneous CAP originating in the eye. The invariant latency, the similarity in waveform of connective potentials to the optic nerve CAP, and the coherent nature of the connective $\mathrm{CAP}$ argues that $\mathrm{C}-\mathrm{Pd}$ and $\mathrm{C}-\mathrm{PI}$ connectives contain axons of pacemaker neurons. Electrical stimulation of C-PI, C-Pd, and PI-Ab connectives all evoked an antidromic CAP in the optic nerve similar to the CAP originating in the eye, again arguing that axons from the eye travel without synaptic interruption through these connectives. The antidromic potential was much larger and easier to record than orthodromic potentials because the optic nerve is a very small nerve that contains only pacemaker and photoreceptor fibers, and the signal-to-noise ratio is therefore much more favorable. Connective stimulation would synchronously activate an antidromic potential in any optic afferents (i.e., photoreceptor as well as pacemaker neuron axons) or efferents in the connective and, thus, does not offer conclusive proof that the antidromic potential represents firing of pacemaker axons. However, the close similarity in waveform between the antidromic CAP, the spontaneous optic nerve CAP, and the orthodromic connective CAPs suggests that this potential reflects activity of pacemaker axons.

The combined results of $\left[{ }^{3} \mathrm{H}\right] \mathrm{DOPA}$ labeling and the recording of an orthodromic CAP in C-PI and C-Pd connectives provides strong evidence that these pathways contain pacemaker axons. The PI-Ab connective also contained [ ${ }^{3} \mathrm{H}$ ]DOPA-labeled fibers, but an orthodromic potential was not detected. However, both $\left[{ }^{3} \mathrm{H}\right]$ leucine and $\left[{ }^{3} \mathrm{H}\right]$ OOPA labeling showed that this pathway contains substantially fewer fibers than the other connectives, and the resultant small CAP could become lost in the base line noise. The recording of a Pl-Abstimulated antidromic optic nerve CAP very similar in waveform to the spontaneous optic nerve CAP and the antidromic potential evoked with $\mathrm{C}-\mathrm{PI}$ and $\mathrm{C}-\mathrm{Pd}$ connectives suggests that this pathway also contains pacemaker axons. These experiments support the anatomical findings that pacemaker neurons send axons to cerebral, pedal, pleural, and possibly abdominal ganglia. Only the buccal ganglia do not appear to receive pacemaker innervation.

Stimulation of AT and PT nerves evoked a volley of short-duration unitary potentials in the optic nerve that have a distinctively different waveform than the optic nerve CAP. Many of these potentials probably result from activation of polysynaptic pathways, since they rapidly fatigue with repeated stimulation. Tactile stimulation of the ATs and PTs is known to initiate unitary optic nerve potentials (Block, 1981), and a polysynaptic optic nerve potential after PT stimulation has been reported (Jacklet, 1980). The unitary potentials in the PT nerve that did not fatigue probably represent stimulation of the HRPand cobalt-identified efferent neurons located along the PT nerve.

In several experiments, a small, slow optic nerve potential similar in waveform to the antidromic optic nerve CAP seen with connective stimulation was recorded during repeated PT nerve stimulation. This result, and the observed small [ ${ }^{3}$ 1]DOPA projection to the PT nerve, suggests that the pacemakers also project along the PT nerve. It was more difficult to detect $\left[{ }^{3} \mathrm{H}\right] \mathrm{DOPA}$ label in other anterior nerves, and an optic nerve CAP was not detected in them. However, a potential from a few small fibers would be difficult to detect in these large nerves. Therefore, the possibility remains that these nerves also contain pacemaker axons.

The organization of the circadian system in Aplysia. This study provides the first description of the anatomical interconnections between an identified circadian pacemaker and the CNS. A summary diagram of this circadian system is shown in Figure 9. An autonomous circadian pacemaker is located in each eye (Jacklet, 1969). The output of the pacemaker is a distinctive CAP recorded from the optic nerve, and the frequency of spontaneous CAPs in darkness exhibits a circadian rhythm. An identified population of electrically coupled pacemaker neurons in the eye is the source of the circadian rhythm in CAP frequency, but photoreceptors as well as pacemaker neurons send axons out of the optic nerve (Jacklet, 1976; Jacklet and Rolerson, 1982; Jacklet et al., 1982). Selective labeling of pacemaker neurons with $\left[{ }^{3} \mathrm{H}\right] \mathrm{DOPA}$ shows that pacemaker axons from each eye project bilaterally to cerebral, pedal, pleural, and most probably abdominal ganglia. Comparisons of $\left[{ }^{3} \mathrm{H}\right] \mathrm{DOPA}$ and $\left[{ }^{3} \mathrm{H}\right]$ leucine tracing do not reveal significant differences in central projections, making the projection of photoreceptors uncertain, but they may overlap over a part of the pathway. The contribution of these types to projections along the AT, PT, and ULAB cerebral nerves is also unclear, but at least the PT projection may contain pacemaker fibers. Anterior and posterior tentacles are important sensory structures, and both are known to contain photoreceptors (Jacklet, 1980). Possibly there is direct pacemaker modulation of sensory receptor sensitivity, as is well known in other systems (see Brady, 1981; Page 1981; Barlow, 1983). Optic efferent neurons located along the PT nerve project to the eye through the optic nerve. They may be responsible for the modulation of optic nerve CAP activity originating in the brain (Eskin, 1971; Luborsky-Moore and Jacklet, 1976a). Other optic efferents, which retrogradely transport $\left[{ }^{3} \mathrm{H}\right] \mathrm{DOPA}$, project to the eye over accessory optic nerves (Olson and Jacklet, 1984; L. M. Olson and J. W. Jacklet, manuscript in preparation). Their function is not yet clear. They may be involved in the modulation of the phase of the ocular circadian rhythm requiring the brain that occurs in the paradigm used by Prichard and Lickey (1981), in which the transition from constant light to constant darkness induces phase shifts.

The demonstration of the projection of pacemaker axons throughout virtually the entire rostral CNS suggests that circadian information may be widely distributed to time and to coordinate physiological and behavioral events. Interestingly, this organization is rather different from what is presently known about the output of the vertebrate circadian oscillator in the SCN of the hypothalamus. In that system, thcre are no long ascending or descending projections. Initial processing of circadian information may take place at the level of the hypothalamus and related structures (Moore, 1983) before being relayed to other brain structures that control the circadian-regulated 


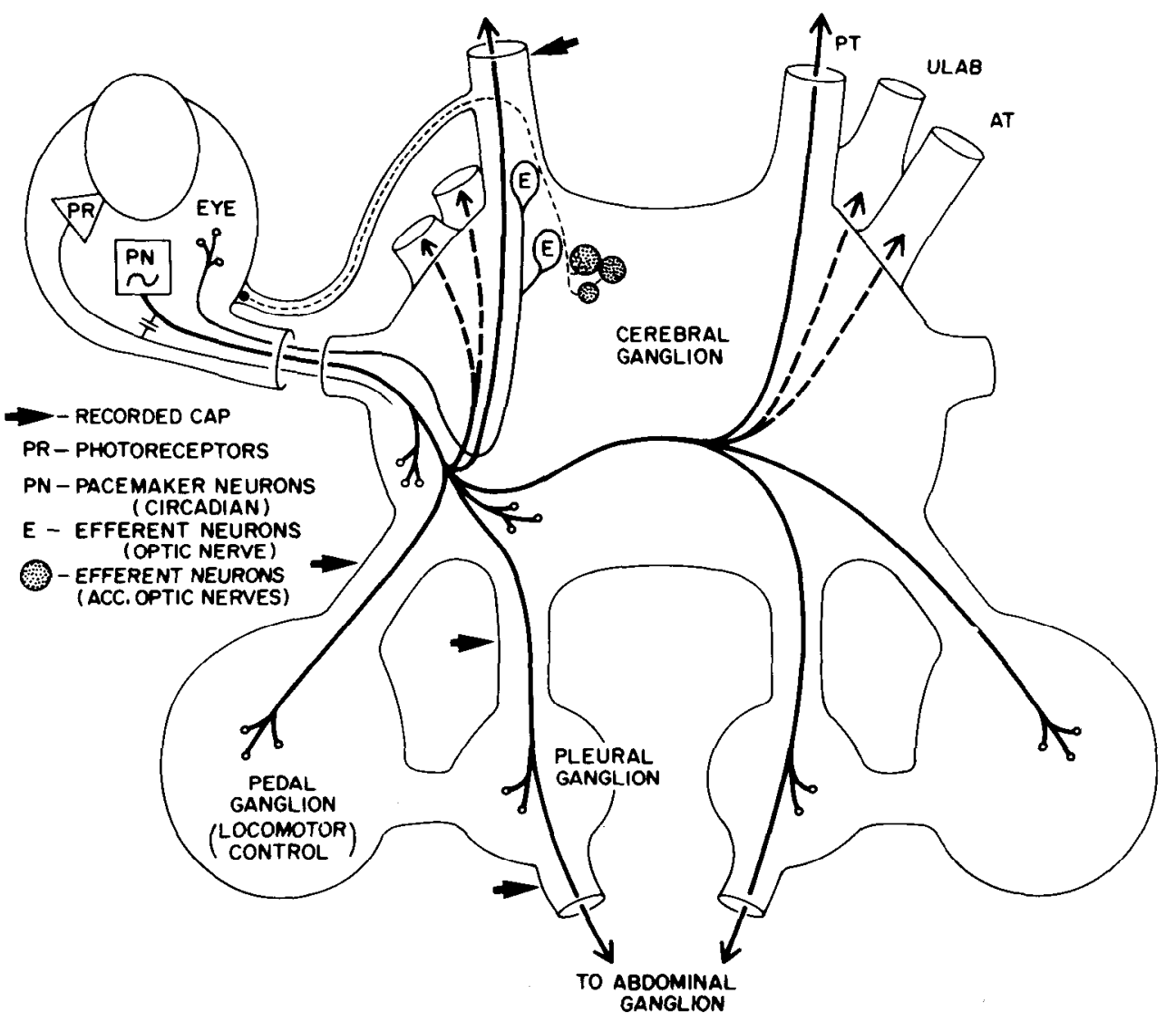

Figure 9. Summary diagram of the circadian system of Aplysia. The electrically coupled population of pacemaker neurons (PN) selectively accumulate and transport tritiated aminergic transmitters and precursors. Transport of radiolabeled material shows that pacemaker neuron axons (heavy lines) project bilaterally to cerebral, pedal, and pleural ganglia, and along the PT nerve. Axons may extend as far as the abdominal ganglion since they project into the PI-Ab connectives. They may also project into other anterior cerebral ganglion nerves (AT and ULAB, heavy dashed lines), which innervate the sensory and motor structures of the head. A coherent orthodromic or antidromic CAP can be recorded from the major connectives and the PT nerves (arrows) which contained $\left[{ }^{3} \mathrm{H}\right]$ DOPA-labeled fibers. Two separate and biochemically distinct populations of neurons project to the eye along the optic nerves and accessory optic nerves. Previously described and HRP-filled efferent neurons $(E)$ project through the optic nerve. A new population of efferents (strippled circles) projected along accessory optic nerves and was shown to ret rogradely transport $\left[{ }^{3} \mathrm{H}\right] \mathrm{DOPA}$. The spc cific projections of the photoreceptors $(P R)$ cannot be distinguished in this study.

behaviors. In Aplysia, the pacemaker axons themselves may carry temporal information directly to the brain centers that mediate behaviors requiring circadian timing. Aplysia may therefore provide a model in which to examine directly the influence of a pacemaker on overt mythmic activities.

The best studied circadian behavior in Aplysia is the circadian regulation of locomotion, which has been extensively documented at the behavioral level (Lickey et al., 1976, 1977; Lickey and Wozniak, 1979). It is known that neural connections between the cerebral and pedal ganglia (Roberts and Block, 1982), as well as between the eye and the cerebral ganglion (Lickey et al., 1976), are essential for the expression of this rhythm. The present study suggests that this connection is the pacemaker neuron axons themselves, which may therefore project directly to locomotor control centers. Neuronal clusters of the network controlling locomotion have been identified on the single-cell level within the cerebral (Fredman and JahanParwar, 1983) and pedal ganglia (Fredman and Jahan-Parwar, 1980), and the pedal ganglia are known to contain the rhythm generator for pedal waves (Jahan-Parwar and Fredman, 1979, 1980). Thus, a cellular analysis of pacemaker input to neurons mediating locomotor activity is possible.

The bilateral and, thus, overlapping projections of the pacemaker neurons from each eye throughout the nervous system are of particular interest. This distribution suggests that a single pacemaker, i.e., a single eye, may be sufficient to direct circadian activities. Furthermore, the overlapping distribution from each eye may provide sites of interaction between the two pacemakers at the level of the driven system. Circadian pacemakers often have been identified in nervous structures that, like the Aplysia eye, are bilaterally present in the nervous system. Behavioral studies have shown that a single pacemaker of the pair is competent to direct circadian activity (Stephen and Zucker, 1972; Page et al., 1977; Lickey et al., 1983; Wiedenmann, 1983); the present demonstration of the extensive bilateral distribution of pacemaker fibers throughout the Aplysia nervous system provides an obvious anatomical substrate for the ability of a single eye to direct circadian behavior. In many organisms redundant pacemakers may normally be coupled, possibly by direct anatomical connections (see Page, 1978; Pickard and Turek, 1982; Roberls and Block, 1983), to ensure that the two pacemakers remain phase locked and that driven systems do not receive conflicting phase signals. However, interactions between pacemakers could conceivably occur at other points in the output of the circadian signal. In fact, work by Page (1983) and Wiedenmann (1983) sugyesis that one pacerriaker may suppress the strength of coupling of the other pacemaker to a driven activity without directly affecting that pacemaker itself (i.e., its phase). In Aplysia, direct coupling between the ocular pacemakers is weak or nonexistent (Hudson and Lickey, 1980). Recently, Jordon et al. (1985) have shown that the Aplysia locomotor rhythm may be stronger with a single eye than when both eyes are intact, which suggests that there may be an interaction between pacemakers and their coupling to driven behaviors. The bilateral and, consequently, overlapping projection of Aplysia pacemaker axons suggests that there may be a functional redundancy in the control by each eye, and provides sites for interactions between the pacemakers or their coupling to driven behaviors.

Communication of pacemaker information. Circadian pacemakers can be thought to act by increasing the probability of an event such as locomotion occurring during one part of the daily cycle and decreasing its probability during another. The widespread dissemination of this modulatory influence to many behavioral and physiological functions suggests that pacemakers may communicate to CNS structures through hormonal or the diffuse, nonconventional synaptic interactions that are characteristic of other systems involved in modulation or setting of behavioral states, such as the aminergic systems involved in arousal. In Aplysia, cutting the optic 
nerves but leaving the eyes in situ is as effective in disrupting circadian locomotor rhythmicity as removing the eyes (Lickey et al., 1976), so that neural activity in the optic nerves, rather than release of substances at the eye itself (Harf et al., 1976), is used for the transfer of circadian information to the neural centers for locomotor control. However, this does nol rule oul a hormonal release at the level of the cerebral ganglion. Such a communication was proposed to occur at the optic nerve/vascular system interface as the nerve enters the cerebral ganglion (Luborsky, 1975). Although in this study we could not distinguish photoreceptor varicosities and pacemaker neuron varicosities in cerebral ganglion neuropil, we were unable to find anatomical evidence for hormonal secretion by any optic afferents at this interface, and no labeled optic profiles were seen to contact obvious vascular or neurohemal spaces in other areas of the projection, although our examination was by necessity preliminary. The identification and characterization of pacemaker terminals may now be resolved with $\left[{ }^{3} \mathrm{H}\right] \mathrm{DOPA}$ electron microscopic autoradiography, given the selective accumulation and transport of $\left[{ }^{3} \mathrm{H}\right]$ DOPA by pacemaker neurons.

HRP-filled axons were found predominantly within neuropil, and many had a varicosc appcarance. In ultrastructural examination, labeled profiles containing small, uniformly distributed electron-lucent vesicles probably represent the varicosities seen at the light level. Throughout most of the examined projection, we rarely encountered putative active zones in which vesicles were aligned along a synaptic membrane. The lack of a well defined active zone appears to be a general feature of Aplysia neuropil (Coggeshall, 1967; Schwartz and Shkolnik, 1981; but see Tremblay et al., 1979). However, varicosities filled with vesicles are thought to be the active zones where synaptic transmission occurs (Bailey et al., 1979, 1981a, b; Schwartz and Shkolnik, 1981). The overall paucity of identifiable active zones in Aplysia makes it difficult to determine whether the HRP-labeled, vesicle-filled optic profiles contain conventional active zones or release their product more diffusely, as is thought to occur in vertebrate aminergic systems, which also lack conventional synaptic profiles. The vesicle-filled varicosities occurred in large numbers in the neuropil, making it likely that information from retinal cells is widely disseminated.

In sharp contrast to the optic ganglion and optic tract midline projection, and in contrast to Aplysia neuropil in general, numerous putative active zones could be identified in the lateral terminus of the optic tract. The lateral terminus forms a compact, glomeruluslike structure of densely packed fibers. Its unusual structure and the uncormmonly larye numbers of active zones indicate that this area is an important site for transfer of ocular pacemaker or photoreceptor information.

The widespread distribution of pacemaker neuron afferents to almost all ganglia in the animal and the large numbers of presumptive synaptic varicosities within neuropil suggest that communication between pacemaker neurons and other CNS elements is mainly through direct synaptic communication and possibly through the semiprivate, modulatory communication suggested for aminergic system (see Dismukes, 1979).

\section{References}

Aghajianian, G: K., and F. E. Bloom (1967) Localization of ${ }^{3} \mathrm{H}$-serotonin in rat brain by electron-microscopic autoradiography. J. Pharmacol. Exp. Ther. 156: 23-30.

Aschoff, J. (1981) Freerunning and entrained circadian rhythms. in Handbook of Behavioral Biology, J. Aschoff, ed., Vol. 4, pp. 81-94, Plenum Press, New York

Bailey, C. H., R. D. Hawkins, and M. C. Chen (1983) Uptake of $\left({ }^{3} \mathrm{H}\right)$ serotonin in the abdominal ganglion of Aplysia californica. Further studies on the morphological and biochemical basis of presynaptic facilitation. Brain Res. 272: 71-81.

Bailey, C. H., R. D. Hawkins, M. C. Chen, and E. R. Kandel (1981a) Interneurons involved in mediation and modulation of gill-withdrawal reflex in Aplysia. IV. Morphological basis of presynaptic facilitation. J. Neurophysiol. 45: 340-360
Bailey, C. H., P. Kandel, and M. Chen (1981b) Active zone at Aplysia synapses: organization of presynaptic dense projections. J. Neurophysiol. 46: 356-368.

Bailey, C. H., E. B. Thompson, V. F. Castellucci, and E. R. Kandel (1979) Ultrastructure of the synapses of sensory neurons that mediate the gillwithdrawal reflex in Aplysia. J. Neurocytol. 8: 415-444.

Barlow, R. B., Jr. (1983) Circadian rhythms in the Limulus visual system. J. Neurosci. 3: 856-870.

Berger, B., and J. Glowinski (1978) Dopamine uptake in serotoninergic terminals in vitro: A valuable tool for the histochemical differentiation of catecholaminergic and serotoninergic terminals in rat cerebral structures. Brain Res. 147: 29-45.

Block, G. D. (1981) In vivo recording of the ocular circadian rhythm in Aplysia. Brain Res. 222: 138-143.

Brady, J. (1981) Behavioral rhythrns in inverlebrates. In Handbook of Behavioral Biology, J. Aschoff, ed., pp. 125-144, Plenum Press, New York.

Carpenter, D., G. Breese, S. Schanberg, and I. Kopin (1971) Serotonin and dopamine: Distribution and accumulation in Aplysia nervous and nonnervous tissue. Int. J. Neurosci. 2: 49-56.

Coggeshall, R. E. (1967) A light and electron microscope study of the abdominal ganglion of Aplysia californica. J. Neurophysiol. 30: 12631286.

Corrent, G., D. McAdon, A. Eskin (1978) Serotonin shifts the phase of the circadian rhythm from the Aplysia eye. Science 202: 977-979.

Dismukes, R. K. (1979) New concepts of molecular communication among neurons. Behav. Brain Sci. 2: 109-118

Eales, N. B. (1921) L. M. B. C. Memoirs, No. XXIV. Aplysia. Trans. Liverpool Biol. Soc., pp. 184-265.

Eskin, A. (1971) Properties of the Aplysia visual system: In vitro entrainment of the circadian rhythm and centrifugal regulation of the eye. $Z$. Vergl. Physiol. 74: 353-371.

Eskin, A. (1979) Circadian system of the Aplysia eye: Properties of the pacemaker and mechanisms of its entrainment. Fed. Proc. 38: 25732579.

Fredman, S. M., and B. Jahan-Parwar (1980) Role of pedal ganglia motor neurons in pedal wave generation in Aplysia. Brain Res Bull. 5: 179-193.

Fredman. S. M., and B. Jahan-Parwar (1983) Command neurons for locomotion in Aplysia. J. Neurophysiol. 49: 1093-1117.

Goldberg, D. J., and J. H. Schwartz (1980). Fast axonal transport of foreign transmitters in an identified neurone of Aplysia californica. J. Physiol. (Lond.) 307: 259-273.

Goldman, J. E., and J. H. Schwartz (1974) Cellular specificity of serotonin storage and axonal transport in identified neurons of Aplysia californica. J. Physiol. 242: 61-76.

Goldstein, R., H. B. Kistler, Jr., H. W. M. Steinbusch, and J. H. Schwartz (1984) Distribution of serotonin-immunoreactivity in juvenile Aplysia. Neuroscience 11: 535-547

Grafstein, B., and D. S. Forman (1980) intracellular transport in neurons. Physiol. Rev. 60: 1167-1283.

Harf, L., S. Arch, and A. Eskin (1976) Polypeptide secretion from the eye of Aplysia californica. Brain Res. 111: 295-299.

Hendrickson, A, and S. B. Edwards (1978) The use of axonal transport for autoradiographic tracing of pathways in the CNS. In Neuroanatomical Research Techniques, R. T. Robertson, ed., pp. 242-291. Academic Press, Inc., New York.

Hudson, D. J., and M. E. Lickey (1980) Internal desynchronization between two identified circadian oscillators in Aplysia. Brain Res. 183: 481-485.

Iversen, L. L. (1975) Uptake processes for biogenic amines. In Handbook of Psychopharmacology, L. L. Iversen, S. D. Iversen, and S. H. Snyder, eds., Vol. 3, pp. 381-442, Plenum Press, New York.

Jacklet, J. W. (1969) A circadian rhythm of optic nerve impulses recorded in darkness from the isolated eye of Aplysia. Science 164: 562-564.

Jacklet, J. (1976) Dye marking neurons in the eye of Aplysia. Comp. Biochem. Physiol. 84: 1-15

Jacklet, J. W. (1977) Neuronal circadian rhythm: Phase shifting by a protein synthesis inhibitor. Science 198: 69-71.

Jacklet, J. W. (1980) Light sensitivity of the rhinophores and eyes of Aplysia J. Comp. Physiol. 136: 257-262.

Jacklet, J. W. (1985) Neurobiology of circadian rhythms generators. Trends Neurosci. 8: 69-73.

Jacklet, J. W. and C. Rolerson (1982) Electrical activity and structure of retinal cells of the Aplysia eye. Il. Photoreceptors. J. Exp. Biol. 99: 381395.

Jacklet, J. W., L. Schuster, and C. Rolerson (1982) Electrical activity and structure of retinal cells of the Aplysia eye. I. Secondary neurons. J. Exp. Biol. 99: 369-380. 
Jahan-Parwar, B., and S. M. Fredman (1976) Cerebral ganglion of Aplysia: Cellular organization and origin of nerves. Comp. Biochem. Physiol. 54A: 347-357.

Jahan-Parwar, B, and S. M. Fredman (1979) Neural control of locomotion in Aplysia: Role of the central ganglia. Behav. Neural Biol. 27: 39-58.

Jahan-Parwar, B., and S. M. Fredman (1980) Motor program for pedal waves during Aplysia locomotion is generated in the pedal ganglia. Brain Res. Bull. 5: 169-177.

Jones, E. G., and B. K. Hartman (1978) Recent advances in neuroanatomical methodology. Annu. Rev. Neurosci. 1: 215-296.

Jordon, W. P., M. E. Lickey, and S. O. Hiaasen (1985) Circadian organization in Ap/ysia: Internal desynchronization and strength of locomotor rhythm. J. Comp. Physiol. 156: 293-303.

Lichtensteiger, W., U. Mutzner, and H. Langemann (1967) Uptake of 5hydroxytryptophan by neurons of the central nervous system normally containing catecholamines. J. Neurochem. 14: 489-497.

Lickey, M. E., and J. A. Wozniak (1979) Circadian organization in Aplysia explored with red light, eye removal and behavioral recording. J. Cornp. Physiol. 13: 169-177.

Lickey, M. E., G. Block, D. Hudson, and J. Smith (1976) Circadian oscillators and photoreceptors in the gastropod Aplysia. Photochem. Photobiol. 23: 253-273.

Lickey, M. E., J. A. Wozniak, G. D. Block, D. J. Hudson, and G. K. Augter (1977) The consequences of eye removal for the circadian rhythm of behavioral activity in Aplysia. J. Comp. Physiol. 118: 121-143.

Lickey, M. E., D. J. Hudson, and S. O. Hiaasen (1983) Circadian organization in Aplysia: Relations between locomotor rhythm and eye rhythms after cutting both, one or neither optic nerves. J. Comp. Physiol. 153: 121-143.

Loh, Y. P., and J. W. Jacklet (1977) Catecholamine and protein synthesis in the eye of Aplysia californica. Comp. Biochem. Physiol. 57C: 159-163.

Luborsky, J. L. (1975) Morphological evidence for an aminergic neurosecretory system in the eye of Aplysia californica. Ph.D. thesis, State of University of New York, Albany, NY

Luborsky-Moore, J. L., and J. W. Jacklet (1976a) Aplysia eye: Modulation by efferent optic nerve activity. Brain Res. 115: 501-505

Luborsky-Moore, J. L., and J. W. Jacklet (1976b) Localization of catecholamines in the eyes and other tissues of Aplysia. J. Histochem. Cytochem. 24: $1150-1158$.

Luborsky-Moore, J. L., and J. W. Jacklet (1977) Ultrastructure of the secondary cells in the Aplysia eye. J. Ultrastruct. Res. 60: 235-245.

Menaker, M., and S. Brinkley (1981) Neural and endocrine control of circadian rhythms in the vertebrates. In Handbook of Behavioral Biology, J. Aschoff; ed., Vol. 4, pp. 243-256, Plenum Press, New York.

Mollenhauer, $H$. (1964) Plastic embedding mixture for use in electron microscopy. Stain Technol. 39: 111-114.

Moore, R. Y. (1983) Organization and function of a central nervous system circadian oscillator: The suprachiasmatic hypothalamic nucleus. Fed. Proc. 42: $2783-2789$.

Olson, L. M., and J. W. Jacklet (1982) Optic nerve projections to central ganglia of Aplysia. Soc. Neurosci. Abstr. 8: 547.

Olson, L. M., and J. W. Jacklet (1983) Identification of circadian clock fibers in the CNS of Aplysia. Soc. Neurosci. Abstr. 9:624.

Olson, L. M., and J. W. Jacklet (1984) Identification of a new population of efferent neurons to the eye of Aplysia with retrograde transport of ${ }^{3} \mathrm{H}$ DOPA. Soc. Neurosci. Abstr. 10: 498

Ono, J. K., and R. E. McCamen (1984) immunocytochemical localization and direct assays of serotonin-containing neurons in Aplysia. Neurnscience 3: 549-560.

Page, T. L. (1978) Interactions between bilaterally paired components of the cockroach circadian pacemaker. J. Comp. Physiol. 121: 225-236.

Page, T. (1981) Neural and endocrine control of circadian rhythmicity in invertebrates. In Handbook of Behavioral Biology, J. Aschoff, ed., Vol. 4, pp. 145-169, Plenum Press, New York.
Page, T. L. (1983) Effects of optic-fract regeneration on internal coupling in the circadian system of the cockroach. J. Comp. Physiol. 153: 353-363.

Page, I. L., P. C. Caldarola, and C. S. Pittendrigh (1977) Mutual entrainment of bilaterally distributed circadian pacemakers. Proc. Natl. Acad. Sci. U. S. A. $74: 1277-1281$

Pentreath, V. W., and M. S. Berry (1978) Radioautographic study of 5 hydroxytryptamine containing nerve terminals in central ganglia of Planorbis corneus: Comparison with other species and characteristics of the serotoninergic nerve terminal. J. Neurocytol. 7: 443-459.

Pentreath, V. W. and G. A. Cottrell (1972) Selective uptake of 5-hydroxytryptamine by axonal processes in Helix pomatia. Nature New Biol. 239: 213 214.

Pentreath, V. W., and G. A. Cottrell (1973) Uptake of serotonin, 5-hydroxytryptophan and tryptophan by giant serotonin-containing neurons and other neurons in the central nervous system of the snail (Helix pomatia). Z. Zell. Mikrosk. Anat. 193: 21-35.

Pickard, G. E., and F. W. Turek (1982) Splitting of the circadian rhythm of activity is abolished by unilateral lesions of the suprachiasmatic nuclei. Science 215: 1119-1121

Pittendrigh, C. S. (1981) Circadian systems: Entrainment. In Handbook of Behavioral Biology, J. Aschoff, ed., Vol. 4, pp. 95-124, Plenum Press, New York.

Pittendrigh, C. S., and S. Daan (1976) A functional analysis of circadian pacemakers in nocturnal rodents. V. Pacemaker structure. A clock for all seasons. J. Comp. Physiol. 106: 333-355.

Prichard, R. G, and M R. Lickey (1981) In vitro resetting of the circadian clock in the Aplysia eye. I. Importance of efferent activity in the optic nerve. J. Neurosci. 1: 835-839.

Reynolds, E. (1963) The usc of lcad citrate at high $\mathrm{pH}$ as an clectron-opaque stain in electron microscopy. J. Cell Biol. 17: 208-212.

Roberts, M. H., and G. D. Block (1982) Dissection of circadian organization of Aplysia through connective lesionis and electrophysiological recording J. Exp. Zool. 219: 39-50.

Roberts, M. H., and G. D. Block (1983) Mutual coupling between the ocular circadian pacemakers of Bulla gouldiana. Science 221: 87-89.

Rusak, B. (1979) Neural mechanisms for entrainment and generation of mammalian circadian rhythms. Fed. Proc. 38: 2589-2595.

Schwartz, J. H. (1979) Axonal transport: Components, mechanisms and specificity. Annu. Rev. Neurosci. 2: 467-504

Schwartz, J. H., and L. J. Shkolnik (1981) The giant serotonergic neuron of Aplysia: A multitargeted nerve cell. J. Neurosci. 1: 606-619.

Shaskan, E. G., and S. H. Snyder (1970) Kinetics of serotonin accumulation into slices from rat brain: Relationship to catecholamine uptake. J. Pharmacol. Exp. Ther. 175: 404-418.

Stephen, F. K., and I. Zucker (1972) Circadian rhythms in drinking behavio and locomotor activity are eliminated by suprachiasmatic lesions. Proc. Natl. Acad. Sci. U. S. A. 54: 1521-1527.

Takahashi, J. S., and M. Zatz (1982) Regulation of circadian rhythmicity Science 217: 1104-1111.

Tremblay, J. P., M. Colonnier, and H. McLennan (1979) An electron microscope study of synaptic contacts in the abdominal ganglion of Aplysia californica. J. Comp. Neurol. 188: 367-390.

Tritt, S. H., P. Lowe, and J. H. Byrne (1983) A modification of the glyoxylic acid induced histofluorescence technique for demonstration of catecholamines and serotonin in tissues of Aplysia californica. Brain Res 259 . 159-162.

Turner, J. D., and G. A. Cottrell (1978) Cellular accumulation of amines and amino acids in the central ganglia of a gastropod mollusc, Planorbis corneus: An autoradiographic study. J. Neurocytol. 7: 759-776.

Wicdenmann, G. (1983) Splitting in a circadian activity rhythm: The expression of bilaterally paired oscillators. J. Comp. Physiol. 150: 51-60.

Zatz, M. (1979) Photoentrainment, pharmacology, and phase shitts of the circadian rhythm in the rat pineal. Fed. Proc. 38: 2596-2601 